\title{
SINVLIO: Using Semantics and Fuzzy Logic to provide individual investment portfolio recommendations Ángel García-Crespo
}

angel.garcia@uc3m.es

Computer Science Department

Universidad Carlos III de Madrid

Av. Universidad 30. Leganés, 28911, Madrid, SPAIN

Phone: +34916249417

Fax: +34916249129

\section{José Luis López-Cuadrado}

joseluis.lopez.cuadrado@uc3m.es

Computer Science Department

Universidad Carlos III de Madrid

Av. Universidad 30. Leganés, 28911, Madrid, SPAIN

Phone: +34 916249117

Fax: +34 916249129

\section{Israel González-Carrasco}

israel.gonzalez@uc3m.es

Computer Science Department

Universidad Carlos III de Madrid

Av. Universidad 30. Leganés, 28911, Madrid, SPAIN

Phone: +34 916249117

Fax: +34 916249129

Ricardo Colomo-Palacios (Corresponding Author)

ricardo.colomo@uc3m.es

Computer Science Department

Universidad Carlos III de Madrid

Av. Universidad 30. Leganés, 28911, Madrid, SPAIN

Phone: +34 916245958

Fax: +34 916249129

\section{Belén Ruiz-Mezcua}

belen.ruiz@uc3m.es

Computer Science Department

Universidad Carlos III de Madrid

Av. Universidad 30. Leganés, 28911, Madrid, SPAIN

Phone: +34 916249968

Fax: +34 916249129 


\section{ABSTRACT}

Portfolio selection addresses the problem of how to diversify investments in the most efficient and profitable way possible. Portfolio selection is a field of study that has been broached from several perspectives, including, among others, recommender systems. This paper presents SINVLIO (Semantic INVestment portfoLIO), a tool based on semantic technologies and fuzzy logic techniques that recommends investments grounded in both psychological aspects of the investor and traditional financial parameters of the investments. The results are very encouraging and reveal that SINVLIO makes good recommendations, according to the high degree of agreement between SINVLIO and expert recommendations.

Keywords: Semantic technologies; fuzzy logic; recommender systems; investment portfolio

\section{Introduction}

Portfolio selection discusses the problem of how to allocate one's capital to a large number of securities so that the investment brings the most profitable return [63]. The concept behind investment portfolios is to combine several different investment targets and so avoid concentrating too much risk in any one target, thus dispersing overall investment risk [9]. Selecting securities and investments is not a simple process, as it requires not only optimizing returns, but more importantly, minimizing potential risks [41]. However, with the vast amount of securities in today's market, it is becoming more and more difficult for investors to scrutinize each and every stock on the market [64]. According to Shyng et al. [70], the process of selecting an appropriate investment portfolio can be divided into two stages. The first step starts with observation and experience and ends with beliefs regarding the future performance of available securities. The second stage starts with relevant beliefs about the future performance of various investment products and ends with the choice of a portfolio.

Individual investors, and sometimes even professional fund managers, often allow their emotions to get in the way of rational investment decision-making [4]. In many cases, this may lead to them making mistakes in investment [9]. From a decision-making point of view, the stock portfolio problem can be divided into two questions [40]:

\section{Which stock do you choose?}

\section{Which investment ratio do you use to allocate your capital to this stock?}

The grandfather of portfolio theory and Nobel Prize-winner Harry Markowitz stated, not without reason, that 'Portfolio selection' uses stocks' historical mean and variance together with the efficient frontier paradigm to construct the optimal portfolio [54]. The aim of this paper is to extend his traditional model by including the psychological preferences and the biases of the investor and, as a consequence of this, improve the categorization of the investor. Based on the studies conducted by Watson [78] and Skinner [72], several recent and important works have examined investor psychology and its influence in investment decision making (e.g.[13]; [16]; [21]; [44]; [45]; [38]; [39]). This paper takes previous works into account and proposes a method which uses ontologies to classify, define and match investors and investments of the application of fuzzy and semantic technologies. 
Another special issue of this paper is Recommender systems. Recommender systems are commonly defined as applications that e-commerce sites exploit to suggest products and provide consumers with information to facilitate their decision-making processes [58]. A recommender system can provide a set of solutions that best fit the user, depending on different factors concerning the user, the objective or the context it is applied in. Such systems can reduce search efforts [49] and provide valuable information to assist consumers' decisionmaking process [65] in order to solve the problem of information overload [46]. According to Porcel and Herrera-Viedma [62], a recommender system could be seen as a decision support system (DSS). Adomavicius and Tuzhilin [2] provide a survey of recommender systems and describe various limitations of current recommendation methods, as well as discussing possible extensions to improve recommendation capabilities and make recommender systems applicable to an even broader range of applications. Financial and investment applications for recommender systems are widely covered in the literature (e.g. [5]; [20]; [56]).

The remainder of the paper is structured in four sections. Section 2 reviews relevant works on risk tolerance, fuzzy logic and portfolio selection. Section 3 provides the theoretical foundations of SINVLIO (Semantic INVestment PortfoLIO), as well as its architecture and implementation. Section 4 describes the evaluation process carried out. Finally, section 5 summarizes the main conclusions of this research and outlines the future research.

\section{Background}

The use of intelligent expert systems in the field of investments has been studied from different perspectives. Valentine presents the advantages of the use of expert systems for finances, and states that the behavior of such systems is controlled by the rules provided [77]: if the rules provided by the expert have an economic orientation, then the expert system will have an economic orientation. In this paper, the aim is to provide a behavioral orientation to portfolio recommendations by means of fuzzy logic and semantic technologies. Other approaches build financial models to forecast stock prices which are based on a combination of methods and techniques [11].

Investor risk tolerance has likewise been studied from a broad number of perspectives. Aspects such as wealth [12], gender [50] or even lunar cycles [19] have been studied for their role in determining risk aversion or risk tolerance. Indeed, the study of the influence of the person's perception on financial behavior has become more important over the last two decades [28].

Personal or professional judgment, heuristics, objective single item question, risk scales or mixed measures are commonly used methods in measuring risk tolerance [28]. Based on previous studies on investor characteristics, Statman [73] affirms that while normal investors are affected by emotions and biases, rational investors are not.

Psychological and biological aspects are influential in the formation of economic preferences [35]. Behavioral finance studies how biases and cognitive errors influence both investors and decision making processes [55]. Indeed, aspects of investor psychology have been widely studied in the literature. Muhammad remarks that psychological biases affect investor behavior and prices [55]. Other authors note that these psychological biases can also lead to systematic errors [3]. Following similar considerations regarding the influence of the psychological aspects on the finance, De Bondt et al. propose a new class of asset pricing model by adding behavioral elements [18]. 
In certain cases, personal investment decisions depend on investor intuition and, as mentioned, biases in judgment may influence the decision-making process. Kahneman and Riepe determine a set of questions and recommendations for investment advisors to take into account the psychological biases of the investor (such as overconfidence, optimism or hindsight) in their recommendations [43]. Grable and Lytton and Grable categorize investors according to their responses to a questionnaire [29][28][30][31]. Sudebar et al. [75] also worked on investor categorization through the application of a set of questionnaires. Shefrin and Statman present a complete review of portfolio theories based on behavioral aspects, and propose a positive portfolio theory [68]. Other research lines explore the classification of the investor from a different point of view. Thus, Roscoe and Howorth interpret charts in order to categorize investors. They distinguish between trend-seekers and pattern-seekers, and whether they trade as a system or an art [66]. From a knowledge management perspective, Cheng et al. implement a financial knowledge management system but they do not take into account the above-mentioned personal aspects [10].

Muhammad [55] suggests implementing regulatory policies to minimize the impact of mistakes deriving from psychological factors. According to this author, on the one hand, it is necessary to take into account the psychological aspects in order to avoid the mistakes deriving from the biases and cognitive errors, and, on the other hand, the risk tolerance and the psycho-social aspects of the investor must be considered in order to provide a portfolio in which investors feel good.

In the area of portfolio generation, the use of genetic algorithms has been studied and applied, as reported in [8]. Abiyev and Menekay [1] combine fuzzy logic in order to represent aspects related to the risk and return of each investment, using genetic algorithms to create portfolios [1]. Genetic algorithms have also been applied to stock prediction [6]. Shipley also works with a model using fuzzy logic in order to represent the relationship between risk and return of the investment [69]. The use of neural networks is a classical approach in portfolio selection [36] and risk assessment [82] , even when combined with fuzzy-logic [79]. However, the use of the psycho-social aspects of the investor combined with these techniques has not been reported in the literature.

Furthermore, there are fuzzy logic approaches that have been developed for portfolio selection. While Yan provides a bifuzzy approach for selecting portfolios with a given degree of risk tolerance [81], Gupta et al. present a hybrid approach for simultaneously considering optimal asset allocation and suitability issues [32]. Ghazinoorya et al. recommend portfolio products based on the concept of portfolio matrices combined with fuzzy logic [26], although this approach does not explicitly consider the psycho-social aspects of the investor. Chen and Hung propose a fuzzy approach based on fuzzy linguistic variables in order to represent expert knowledge of portfolio selection [6]. These authors use the investor's risk preferences to determine the investment ratio for each stock in the proposed portfolio. Despite taking into account the risk profile of an investor, this interesting proposal does not determine it.

The decision rules regarding investment selection must be based on a shared vocabulary in order to categorize products from different sources. Ontologies are "a formal and explicit specification of a shared conceptualization" [74]. Ontologies and semantic technologies have 
been widely used over the last years in many intelligent applications that combine ontologies with reasoners (e.g. [23],[22]). Financial Ontologies like FEF ontology [42] or the ontology of the SONAR project [25] have been applied to categorize and recommend financial products. However, ontologies regarding the psycho-social aspects of investors have not been found in the research work carried out so far. The aim of the system proposed in this paper is to combine the semantic approach with fuzzy logic techniques in order to:

- Categorize investors according to their risk tolerance by using an ontology.

- Categorize the investments based on a Financial Ontology.

- Define fuzzy rules according to the semantic descriptions of the investors and the investments (and thus select investments based on an investor's risk tolerance)

- Recommend an optimal portfolio according to the investor risk tolerance

\section{SINVLIO: Architecture and Implementation}

Most pieces of research which explore knowledge representation centre on the characteristics of the portfolio. For example, Shyng et al. [71] classify the personal investment portfolios as conservative, moderate and aggressive; they establish the main categories of each one and highlight the main factors related to these types of investment. Our aim in this paper is to consider the investor's perspective and the emotions he or she may use in selecting the portfolio. As previously mentioned, psychological biases affect investor behaviour and prices [55] and, as a consequence, dealing with investor's psychological biases is a complex problem for investment advisors. Decisions based on subjective elements rather than objective elements usually lead to bad investments and bad results.

If it were possible to predict the stock markets with full accuracy, then investors would always obtain profits. However, great opportunities for large profits are related to high risk levels. As mentioned, investor risk tolerance is a major factor in portfolio selection because there is no guarantee that the investment will bring a return in profits (or the expected degree of profit).

This paper proposes an intelligent financial advisor, based on fuzzy logic and semantic technologies, which is able to make recommendations based on the investor's point of view. The proposed system will recommend the portfolio most suitable to the characteristics of the investor, taking into account his or her preferences and risk tolerance. The main steps of the proposed recommendation process are:

1. Investor categorization.

2. Determining the risk tolerance of the investor category.

3. Investment (portfolio) classification.

4. Matching the portfolio characteristics with the investor categories.

5. Obtaining the recommendation.

Characteristics of both investors and portfolios are represented by means of two domain ontologies, and the relationship between the investor profile and the desirable characteristics 
of the portfolio are represented by means of fuzzy rules. The following subsections detail each of the proposed steps.

\subsection{Investor Categorization}

SINVLIO aims to provide portfolio recommendations based on the investor profile. The starting point of the proposed system is the categorization of the investor. For this categorization, fuzzy logic will be used. The fuzzy logic systems can work effectively with many parameters and nonuniform variables suggesting that they can deal with most of the drawbacks of more conventional techniques. The different characteristics to be 'fuzzified' have been obtained from the literature, and are based on two different perspectives, as shown below.

The first of these parameters hold certain attributes such as gender, age or marital status. All of them are related to risk tolerance [29]. The second perspective sustains that psychological aspects of the investor should be considered in order to determine the investor's risk tolerance [30][31]. Both aspects can be obtained by means of a questionnaire based on the work of Grable and Lytton [30][31]. Once the investor has answered the questionnaire, the system has to determine the investor profile with respect to the risk tolerance. For this purpose, an ontology has been developed in order to represent the characteristics of the investor. On the one hand, this ontology represents the generic attributes of the investor (Figure 1 and Figure 2 ), such as gender, income or marital status. On the other, the psychological aspects are represented by means of specific concepts, like self-esteem, emotion during risk, etc., (Figure 3 and Figure 4), according to the study by Sudebar et al. [75], Grable [29][28] and Grable and Lytton [30][31]. The characteristics of these questionnaires have been tested in real scenarios and promising results have been obtained by these researchers.

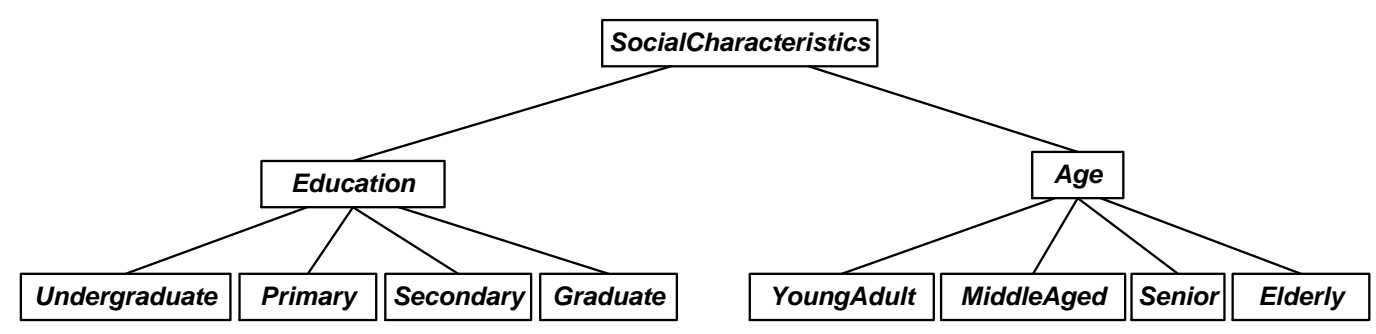

Figure 1. Investor Ontology - Social characteristics (Partial View - 1)

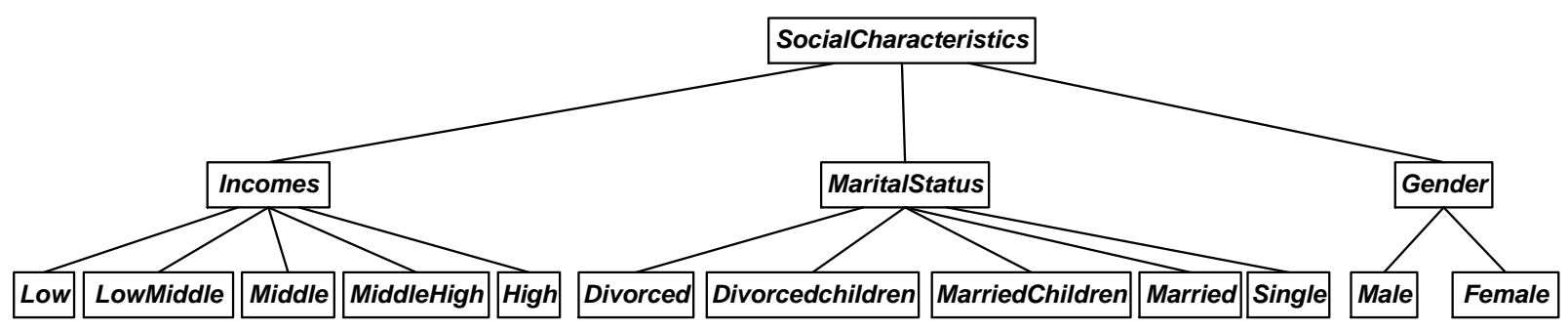

Figure 2. Investor Ontology - Social characteristics (Partial View - 2) 


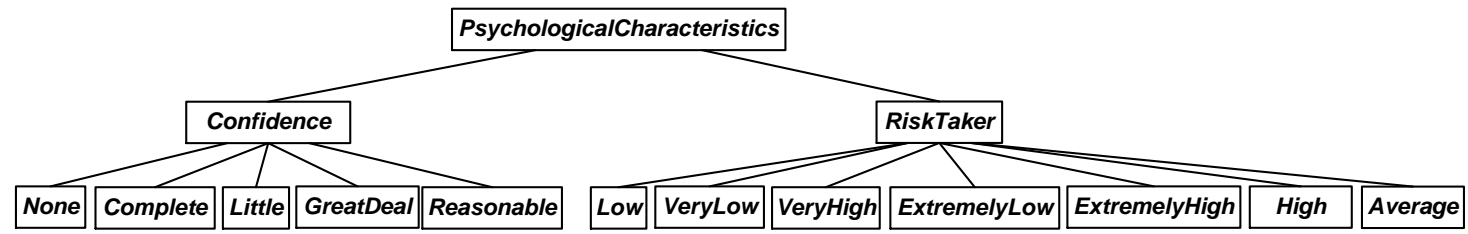

Figure 3. Investor Ontology - Psychological characteristics (Partial View - 1)

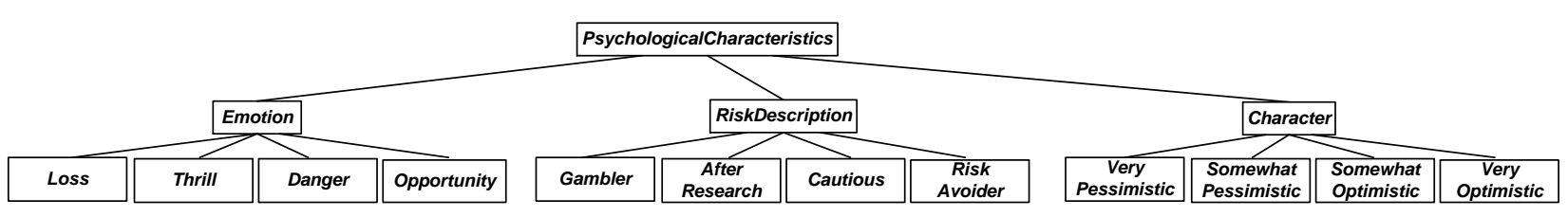

Figure 4. Investor Ontology - Psychological characteristics (Partial View - 2)

\subsection{Risk tolerance determination}

The knowledge required to evaluate investor risk tolerance implies the subjective point of view of an investment advisor. For example, Grable and Lytton establish a score for each response in a questionnaire and the sum of all the scores determines the investor profile [30]. The categorization process is highly subjective and it is difficult to exactly determine the category for a given investor. For this reason, the previously mentioned categories have been 'fuzzified' in order to ease the expression and determine the rules of membership based on the expertise of an investor advisor.

In short, the fuzzy sets theory provides a framework for the representation of the uncertainty of many aspects of human knowledge. Nowadays, fuzzy rule based systems have been successfully applied to a wide range of real-world problems from different areas [33] and in many real-world applications. Although a system can be defined mathematically in a general sense, a fuzzy logic system representation is still preferred by engineers [51].

By means of SINVLIO, investors will be characterized according to their tolerance for risk, depending on their social and psychological characteristics. This will create a set of fuzzy rules to define the investor Risk Tolerance ( $\mathrm{RI}_{\mathrm{Inv}}$ ) from two clusters of variables: Social Behavior (SB) and Psychological Behavior (PB). Both SB and PB have been adapted from the research work mentioned above. These sets of variables were defined from the characteristics obtained through the questionnaires. Two examples of these sets are depicted below:

(1) $\mathrm{RI}_{\mathrm{Inv}}=\mathrm{SB}$ \{age, incomes, marital status, gender and education $\}$ AND PB \{confidence, character, risk taker, emotion on risk, risk description $\}$

Some of the variables of the prior SB and PB clusters could be characterized by fuzzy values, which would lead to a fuzzy set for each of the variables. Therefore, in the design and implementation of a fuzzy logic system, the option exists to choose which of the three most popular membership functions to use: triangular, Gaussian or trapezoidal function [57]. In this paper, the triangular and trapezoidal membership functions have been used for the fuzzy sets. 
Using triangular and trapezoidal functions means that the performance rate will be very fast, although the level of accuracy will be lower than with either of the membership functions [80].

Focusing on the SB cluster, the membership function of the fuzzy variables age and incomes, shown in Figure 5, are adapted from [33] and [15] respectively. In these fuzzy sets, the labels and values for each one are obtained from the cited questionnaires. The Age and Income variables have been defuzzified because they have continuous numerical values. The domain of discourse associated with the fuzzy sets of the SB cluster has been adapted from [75] and is as follows: Defensive, Conservative, Moderate and Aggressive. The rest of the variables of the SB cluster (marital status, gender and education) used to characterize the investor are categorical and they do not need to be fuzzified.

The Psychological variables of the PB cluster, like sensation seeking and self-esteem, have linguistic labels and have been defuzzified to obtain numerical values. The universe of discourse associated with the PB fuzzy sets for psychological risk aversion has been adapted from that proposed by Grable and Lytton [31] to fit with the SB fuzzy sets: Defensive (Low), Conservative (Below-average), Moderate (Above-average) and Aggressive (High).

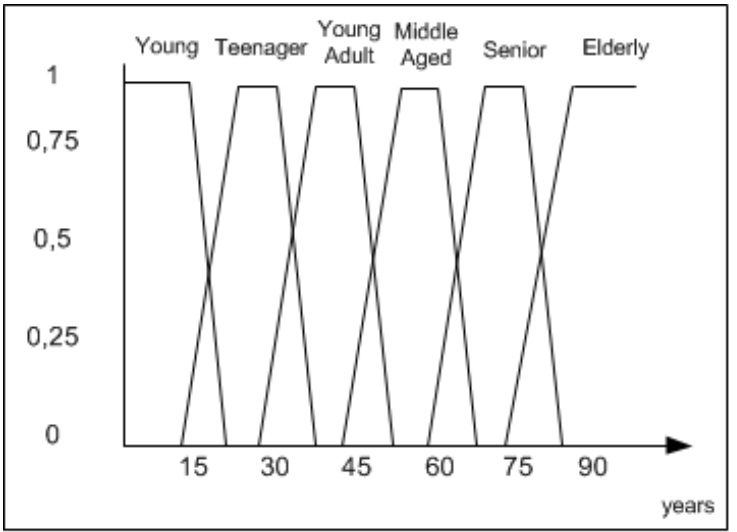

Age Membership function

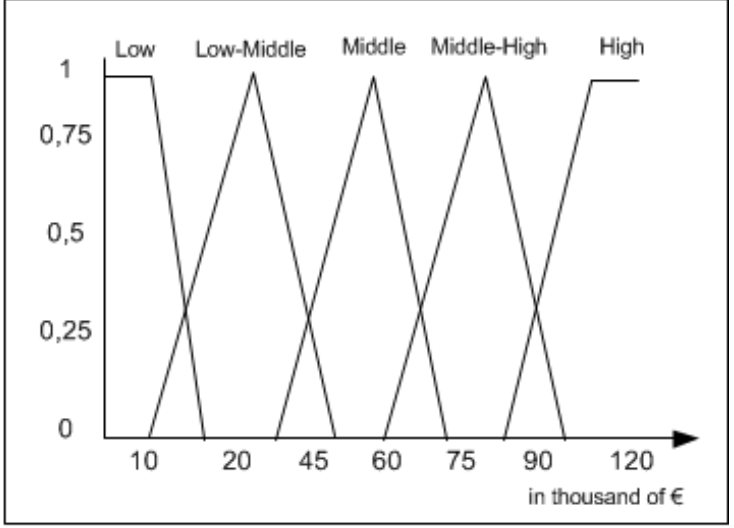

Incomes

Membership function

Figure 5. Age membership function

The notation for the fuzzy sets was defined by Lotfi Zadeh [83]; let $A$ be a fuzzy set defined in the universe $\mathrm{U}$ :

$$
\text { (2) } A=\left\{\left(\mathrm{x}, \mu_{\mathrm{A}}(\mathrm{x})\right) / \mathrm{x} \in \mathrm{U}\right\}
$$

This fuzzy set in the universe of discourse $U$ is characterized by a membership function $\mu A(x)$ taking values in the interval [0.1], and can be represented as a set of ordered pairs of an element $x$ and its membership value to the whole. After defining the fuzzy sets, the fuzzy inference rules may be used to represent the relation between these fuzzy sets. In this context, the fuzzy reasoning process is based, on the one hand, on making inferences from facts and fuzzy relations; and on the other, on a fuzzy combination of evidence which updates the accuracy of beliefs. SINVLIO employs a Mamdani-type fuzzy rule recommender system [52][53], because it is widely accepted and suited to capturing expert knowledge. These fuzzy rules, defined using a set of IF-THEN rules or Bayesian rules, are expressed as follows: 
(3) $\quad \mathrm{Rm}$ : IF $u_{1}$ is $A_{1}{ }^{m}$ AND $u_{2}$ is $A_{2^{m}}$ AND .... $u_{p}$ is $A_{p^{m}}$, THEN $v$ is $B^{m}$

With $\mathrm{m}=1,2, \ldots, \mathrm{M}$, where $\mathrm{A}_{\mathrm{i}}^{\mathrm{m}}$ and $\mathrm{B}^{\mathrm{m}}$ are fuzzy sets in $U_{i} \subset \mathfrak{R}$ (real numbers) and $V \subset \mathfrak{R}$ respectively, $\mathrm{u}=\left(\mathrm{u}_{1}, \mathrm{u}_{2}, \ldots, \mathrm{u}_{\mathrm{n}}\right) \in \mathrm{U}_{1} \times \mathrm{U}_{2} \times \ldots \times \mathrm{U}_{\mathrm{n}}$ and $\mathrm{v} \in \mathrm{V}$, and $\mathrm{x}=\mathrm{x}_{1}, \mathrm{x}_{2}, \ldots, \mathrm{x}_{\mathrm{n}} \in \mathrm{U}$ and $\mathrm{y} \in \mathrm{V}$ are specific numerical values of $u$ and $v$, also respectively. $A$ rule of this type expresses a relation between the sets $A$ and $B$, whose characteristic function would be $\mu_{A} \rightarrow_{B}(x, y)$, and represents what is known as logical implication.

Having defined the main fuzzy theory, this has been applied in SINVLIO following the next steps. First, a number of fuzzy rules to socially describe investors have been identified. Taking into account the number of variables of the SB and PB sets, a wide number of rules are generated. An example of these types of rules to define the aggressive investors according to their social characteristics would be:

(4) IF age is young AND gender is male AND marital status is single THEN investor is Socially-Aggressive

It is generally believed that younger investors take greater risks in anticipation of higher returns. With growing age, they rebalance portfolio in favor of safer and more secured (though somewhat lower) returns. In finance literature, it is believed that women invest more conservatively and are less likely to hold risky assets than men. They do not rebalance their portfolios frequently and prefer a buy-and-hold strategy. Finally, there are marital-status influences on consumption, savings and investment behavior.

The psychological fuzzy rules are similar and define each investor within the same domain. For example:

(5) IF EmotiononRisk is thrill AND confidence is complete AND character is optimistic THEN investor is Psychologically-Moderate

To be able to get the full set of social rules that model this problem, all theoretically possible combinations of $\mathrm{P}_{\mathrm{t}}$ rules were considered, taking into account the number of antecedents $p$ and the number of input fuzzy sets $A_{p}$ considered for each antecedent. Thus, for each consequent, the theoretical number of possible rules is:

(6) $\quad \mathrm{P}_{\mathrm{t}}=\prod_{n} A_{n}$ for $\mathrm{n}=1 \ldots . \mathrm{p}$;

Also, traditionally linguistic labels have been used as modifiers of fuzzy sets, equivalent to what would be the natural language adverbs. The interpretation in the fuzzy model of these statements is the composition of the membership function with a simple arithmetic operation. For example, it is usual to consider the square of the original membership function as an interpretation of the adverb "very":

$$
\mu_{\text {very } \mathrm{A}}(\mathrm{x})=\left(\mu_{\mathrm{A}}(\mathrm{x})\right)^{2}
$$


With these new linguistic labels, 'very', 'highly', 'regular' (not labeled), 'some' and 'a bit' (sorted by relevance), there will be a fine-tuning of the categorization of risk an investor takes, both at a social and psychological level.

After that a matrix to gather the social and psychological factors of the investor was constructed. The motivation of this matrix is the Business Portfolio Analysis Matrix [17][76], a tool that uses quantified performance measures and growth targets to analyze a firm's business units (called strategic business units, or SBUs, in this analysis) as though they were a collection of separate investments. In this proposal, the vertical axis represents the social behavior (instead of the market growth rate) and the horizontal axis symbolizes the psychological behavior (instead of the relative market share). Following the position of the labels in the business portfolio analysis (stars, cash cows, question marks and dogs), the labels for the SB and PB sets are settled in the matrix based on its relevance. The labels stars, cash cows, question marks and dogs, are adopted from the Boston Consulting Group (BCG) matrix where they are used to rank the business units or products [34]. The BCG matrix is a very widespread and useful tool for allocating resources and it is employed as an analytical tool in several companies.

Following the basis of the BCG matrix, the Business Portfolio Analysis Matrix gives a series of quadrants that locate the investor's risk tolerance within these two dimensions. The fine-tuning (performed by the linguistic adverbs) can move the location of an investor within the same quadrant, bringing it closer to its adjacents. For the following investor categorization "highly Socially-Aggressive and some Psychologically-Moderate" the graphical example of this behavior is the following:

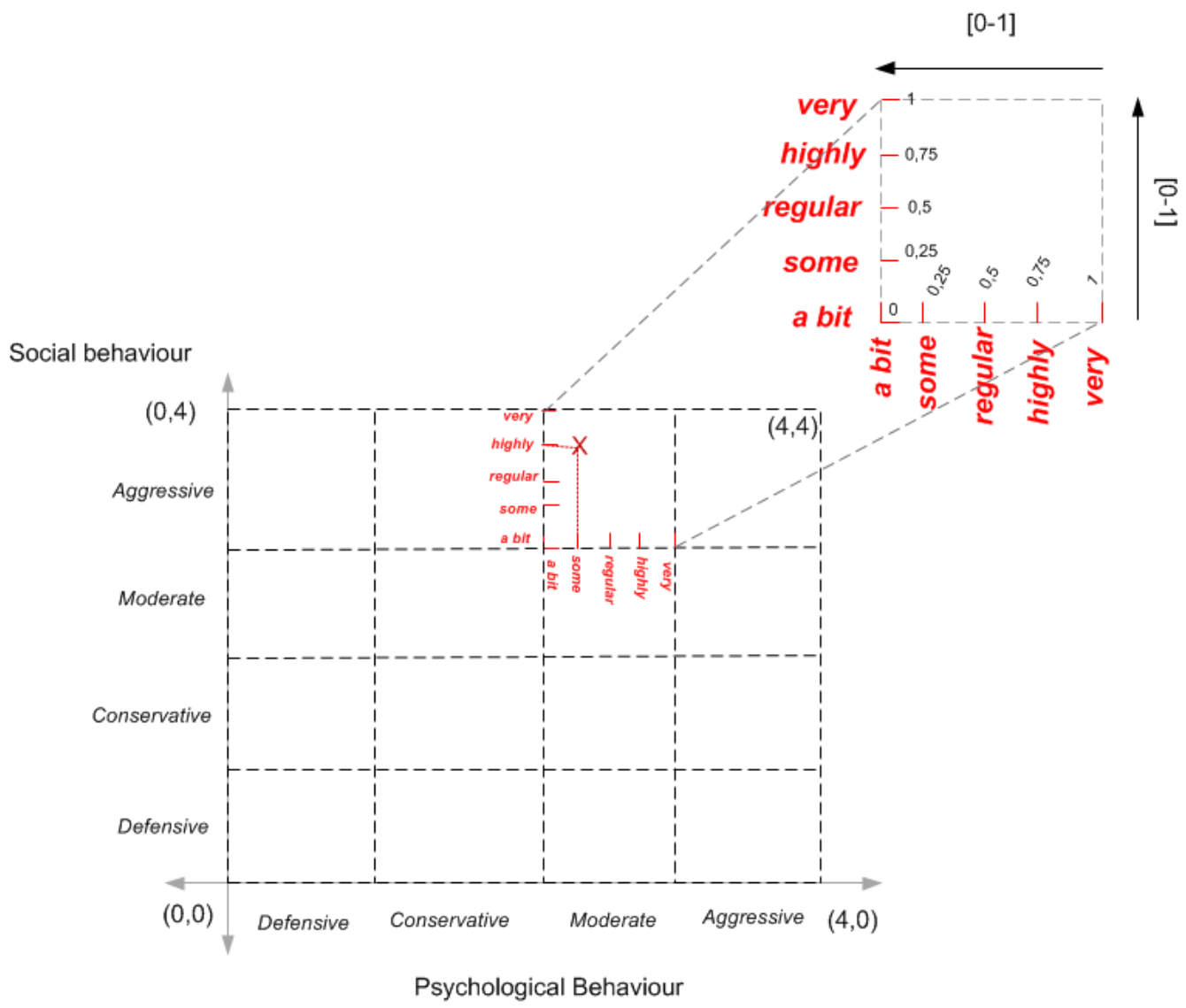

Figure 6. Bi-dimensional matrix for investor behavior 
Each quadrant is divided into sections that correspond to the linguistic labels explained previously. The numerical conversion of these labels can be seen in Figure 6, and it corresponds with a value in the range [0-1].

\subsection{Investment Categorization}

Once the investor has been fully categorized, the next step consists of categorizing the products in the portfolio. In order to do this, it is necessary to consider a set of similar characteristics for all the products. The characteristics of each product have been adapted from the FEF ontology [42] and the taxonomy of Gupta el al. [32]. Each financial product is thus labeled according to the common vocabulary defined by the ontology depicted in Figure 5.

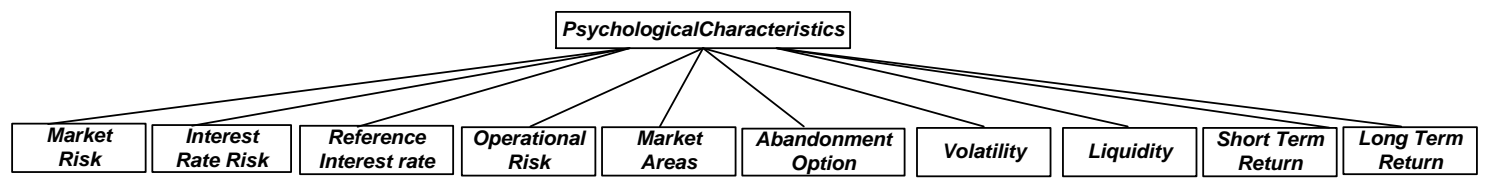

Figure 7. Portfolio Investment Ontology.

Figure 7 shows the ontology developed in order to represent the characteristics of the investment, such as volatility, liquidity, market area or market risk. Based on these characteristics, it is necessary to determine which financial products fit with the risk tolerance of the investor. To achieve this, each investment is classified according to expert knowledge. The variables available to describe the investment are: market risk, interest rate risk, reference interest risk, operational risk, liquidity, volatility, short term return and long term return (all broken down with the labels Low, Average and High). The abandonment option is labeled with true or false and, finally the market area is divided into capital markets, commodities, derivates, fixed income and future options.

The characteristics of the financial products in the portfolio will be categorized with linguistic labels using fuzzy sets according to the some terms employed in the investor categorization: Defensive, Conservative, Moderate and Aggressive.

Research into portfolio recommendation has centered on financial aspects instead of psychological aspects of the investor. However, investor feeling about the investment is a key factor in satisfying investor expectations. The 'fuzzification' of the rules of financial product selection is based on the concept of matrices as well as a set of fuzzy rules to describe portfolio investment. These rules have been obtained with the expertise of an investor advisor for Spanish investors. Examples of these types of rules, which define an aggressive investment according to its characteristics, would be:

(8) IF Liquidity is high AND Market Area is Capital Markets AND Volatility is normal THEN investment is Socially-Moderate and Psychologically-Conservative

(9) IF Market Risk is low AND Liquidity is low AND Market Area is fixed income AND Long Term Return is normal THEN investment is Socially-Defensive and Psychologically-Defensive 
The first rule corresponds to the shares of an important bank in the IBEX 35 market and the second to German bonds, always from the viewpoint of Spanish investors. The linguistic labels, 'very', 'highly', 'regular' (not labeled), 'some' and 'a bit', allow a fine-tuning in the categorization of investment risk.

The variables Market Area (capital markets, commodities, etc.), Abandonment Option (yes or no), Operational Risk (high, medium or low), Interest Rate Risk (high, medium or low) and Market risk (high, medium or low) are not fuzzy because they have categorical values. The membership functions for the remaining investment variables have been defined by the expert, and the fuzzy sets obtained are shown in Figure 8.

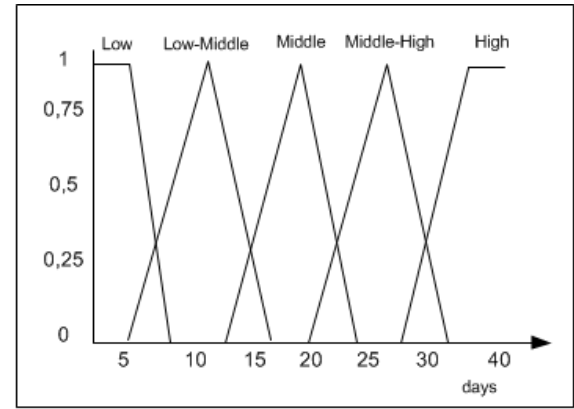

Liquidity Membership

function

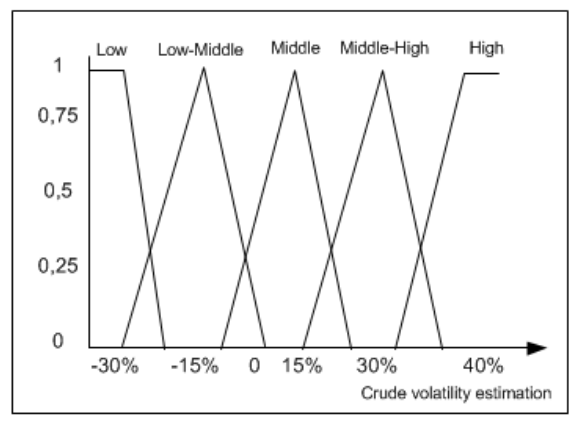

Volatility Membership function
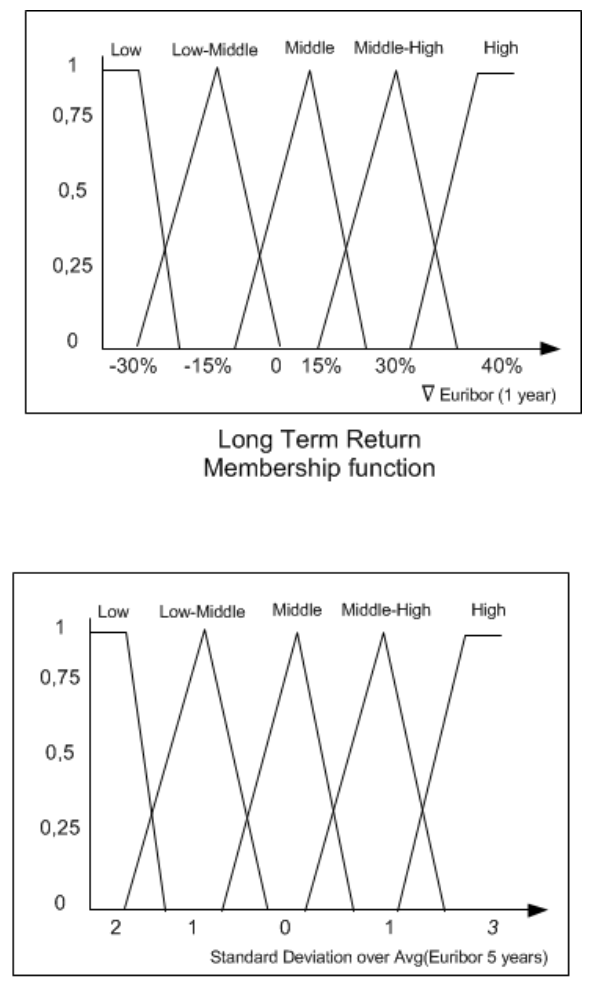

Reference Interest Rate Membership function

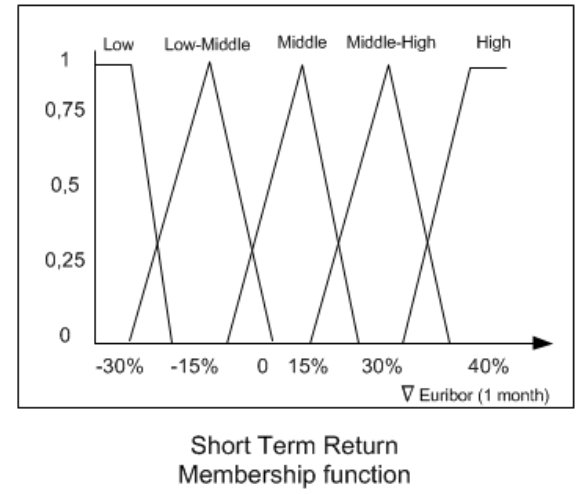

Figure 8. Membership functions for the fuzzy investment variables 
Finally, a matrix has been constructed which represents the fuzzy investment categorization based on the social behavior (vertical axis) and the psychological behavior (horizontal axis) of the investor (see Figure 9). This provides a series of quadrants that enable each investment to be located according to the investor's risk tolerance. As well as this, the fine-tuning (performed by the linguistic adverbs) can move the location of an investment within the same quadrant, bringing it closer to its adjacents. An example of this behavior can be observed by means of the following investment (investment 2 in Figure 9iError! No se encuentra el origen de la referencia.): some Moderate (social) and regular Conservative (psychological).

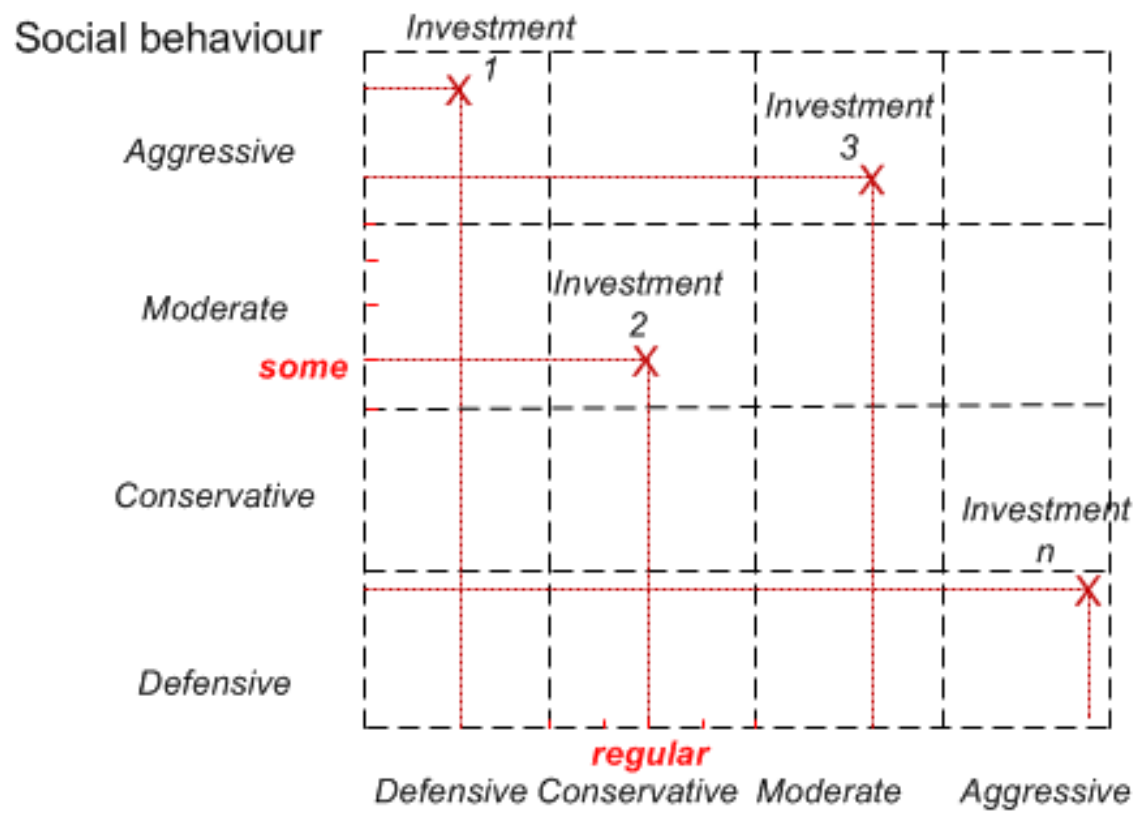

Psychological Behaviour

Figure 9. Fuzzy portfolio matrix based on investor's risk tolerance

\subsection{Matching and Recommendation}

The approach explained in the previous sections only allows recommendations in the same quadrant. Hence, and taking into account the research of Ghazinoorya et al. [26], a series of geometric concepts will be used to define areas of influence in the matrices around each investor or investment. This means that an investor or investment can form relationships with various quadrants in each matrix, allowing greater freedom of choice for the recommendation system.

To obtain the figure associated with the area of influence, the system will calculate the possible shift or offset, in each of " $x$ " and " $y$ "axis, from the point in which the investor or investment is located within the fuzzy matrix. Therefore, the area of influence appears as an ellipse whose horizontal axis corresponds to the psychological characteristics and the y-axis with the social ones. The ellipse is characterized by the point where investment or the investor is located within a quadrant $\left(x_{0} . y_{0}\right)$ and the two semi-axes that correspond to the freedom of 
decision (FD) in the social and psychological direction. The offset associated with this equation is as follows:

$$
\begin{aligned}
& F D_{\text {Investor/Invesment }}=\left(\text { off }_{\text {set }}, \text { off }_{\text {set }}\right)=\left(\text { Eval }_{P_{\text {syco }}, \text { Eval }_{\text {Social }}}\right)= \\
& \left(\frac{\sum_{l=1}^{\mathrm{n}} \mathrm{w}_{i}}{n}, \frac{\sum_{j=1}^{\mathrm{h}} \mathrm{w}_{j}}{\mathrm{k}}\right)
\end{aligned}
$$

where $\mathrm{i}$ and $\mathrm{j}$ are the social psychological characteristics. The geometrical fine-tuning to move inside a quadrant within the range $[0,1]$ is obtained by using the values of the weights $W$. These values, for each Psychological and Social variable, are determined by the expert by a trial and error process. For example, from the rules (4) and (5) the offset ${ }_{x}$, and offset $t_{y}$ will be assessed only for the columns Socially-Aggressive and Psychologically-Moderate, based on the weights associated with the correct values of the characteristics of the investor and investment. Then, the area of influence around each investment or investor in the matrices will be calculated using an ellipse with center $\left(\mathrm{x}_{0} . \mathrm{y}_{0}\right)$, horizontal deviation ( $\mathrm{x}_{0}$ - offset $\mathrm{x} \mathrm{x}_{0}+$ offset $\left.\mathrm{x}^{\prime}\right)$ and vertical deviation ( $y_{0}$ - offsety, $y_{0}+$ offsety'). For the ellipse associated with the area of influence used the equation of an ellipse in Cartesian coordinates with center at point $\left(\mathrm{x}_{0} . \mathrm{y}_{0}\right)$ :

$$
\frac{\left(x-x_{0}\right)^{2}}{a^{2}}+\frac{\left(y-y_{0}\right)^{2}}{b^{2}}=1
$$

where $a>0$ and $b>0$ are the semiaxes of the ellipse (a corresponds to the $x$-axis, $b$ the $y$-axis) and correspond to the offset on the $x$ axis and the offset on the $y$-axis respectively. Figure 10 shows the areas of influence on the fuzzy matrices for an investor and an investment.

Investor Categorization

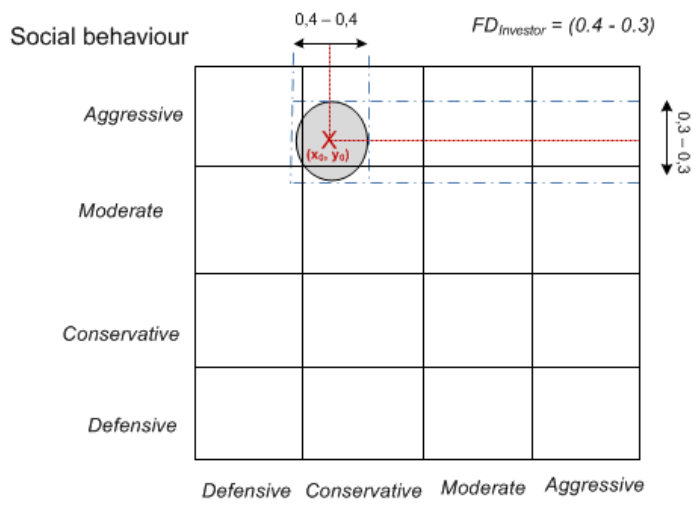

Psychological Behaviour
Investment Categorization

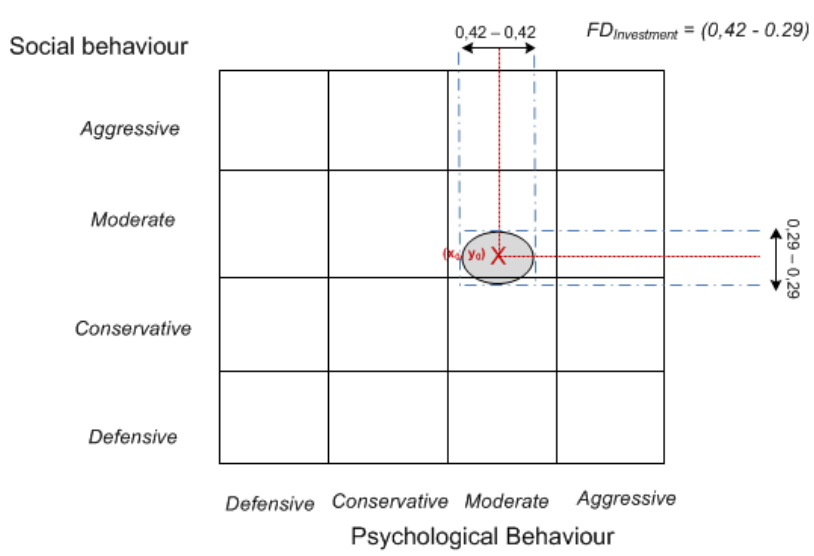

Figure 10. Geometrical approximation for the area of influence in investor and investment matrix

To find the recommendation of products portfolio that best fits the characteristics of an investor, they are both represented by means of a matrix structure (Figure 10). In such a matrix structure, columns represent the psychological behavior and the rows represent the social behavior. The product will be represented in this matrix structure in the form of ellipses. Investors will also be represented according their social and psychological characteristics. In this way, both investors and investments are plotted into the matrix according to their 
characteristics. As shown in Figure 11, once the ellipses for representing investments and investors have been plotted, they can overlap each other. This overlap, along social and psychological lines, will occur between areas of influence of both products and investors (Figure 11). The more overlapped area between an investment and an investor, the more suitable the investment for that investor. If investment ellipses do not overlap the investor ellipse then the suitability of the investment can be measured by means of the distance between ellipses: the nearer the investment, the more suitable it is for the investor.

\section{Example 1}

Social behaviour

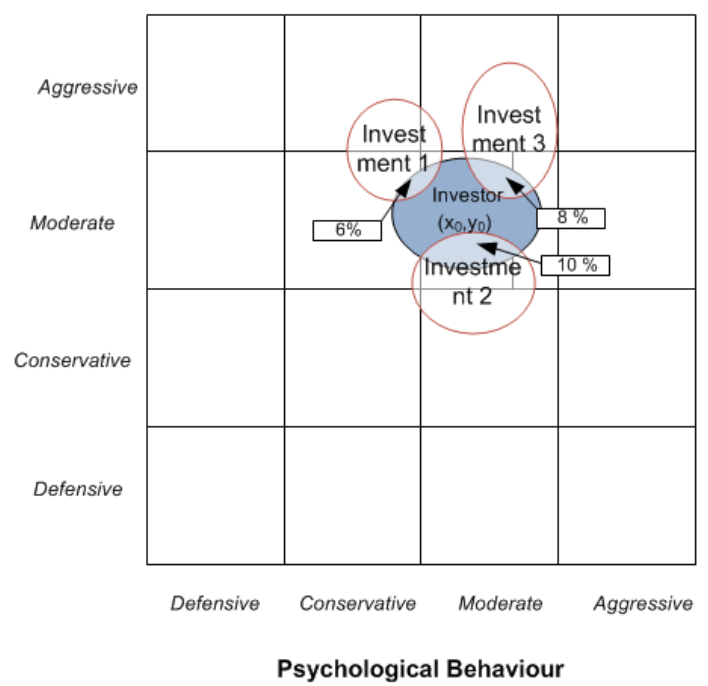

Example 2

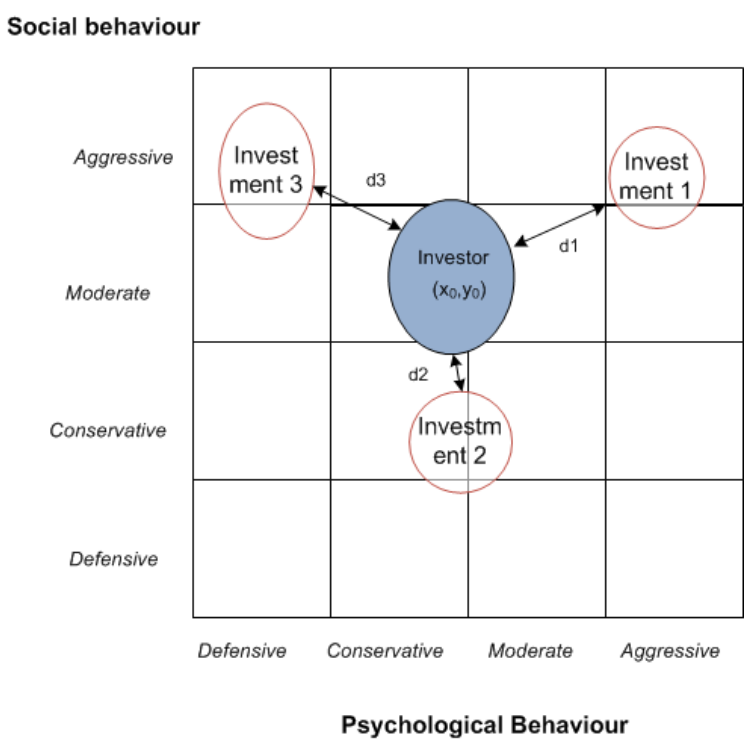

Figure 11. Different geometrical recommendations with priority

To prioritize the recommendations of the portfolio, it needs to be determined whether or not there is an overlap between the areas of influence of the investor and the investment. In the first instance, the priority is defined according to the percentage of overlap of each product with the investor: a higher percentage means a higher priority. In the second instance, in the absence of overlap, the distance between the different ellipses of investment and the investor will be calculated: a shorter distance implies a highest priority. In this case, the minimum distance is defined following the equation of Euclid:

$$
\sqrt{(x-a)^{2}+(y-b)^{2}}
$$

where $(x, y)$ denotes a generic point of the ellipse of the investor and $(a, b)$ a generic point of the ellipse of the investment. Thus, the expression associated with the minimum distance by the partial derivatives of each variable must be minimized:

$$
f(x, y, a, b)=(x-a)^{2}+(y-b)^{2}=f^{\prime}(x)=d x f\left\{\begin{array}{l}
f^{\prime}(x)=d x f(x, y, a, b) \\
f^{\prime}(y)=d x f(x, y, a, b) \\
f^{\prime}(a)=d x f(x, y, a, b) \\
f^{\prime}(b)=d x f(x, y, a, b)
\end{array}\right.
$$


where the points where $f(x, y, a, b)$ reaches extreme values are the solutions of the proposed system.

\subsection{Use case}

For a better understanding of the proposal, a numerical example has been included in this section. The characteristic of the investor Peter Smith for the SB and PB sets are the following:

$R I_{I n v}=($

$S B=\{$ Age $=$ Middle aged, Incomes $=$ Middle, Marital status $=$ Married with Children, Gender $=$ Male, Education $=$ UnderGraduate $\}$

AND

$P B=\{$ Confidence $=$ Reasonable Amount, Character $=$ SomeWhatPesimistic, Risk taker $=$ Average, Emotion on risk $=$ opportunity, risk description $=$ cautious $\}$

)

Based on the fuzzy rules defined by the expert and the linguistic adverbs, the resulting categorization of the investor is:

(1) The investor Peter Smith is Regular Socially-Moderate and Highly PsychologicallyModerate

On translating this rule to the business portfolio analysis matrix, the following representation is obtained:

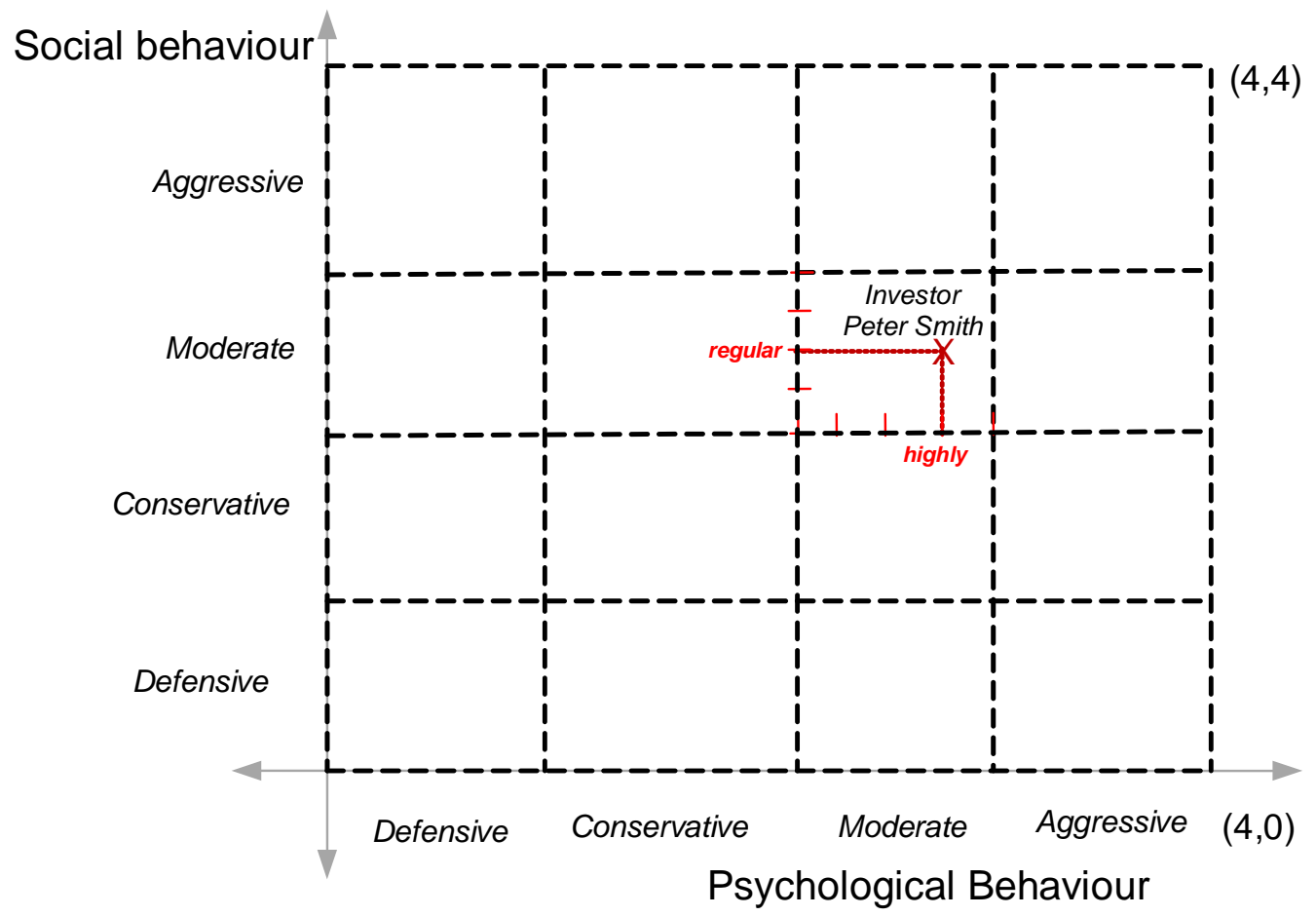

Figure 12. Matrix representation of the investor Peter Smith. 
The investments selected for this numerical simulation are both Structured Deposits, and they are labeled as Investment A and Investment B. Taking into account the variables available for these investments, Table 1 shows the categorization obtained.

\section{Variable Investment A Investment B}

\begin{tabular}{|l|l|l|}
\hline Market Risk & Medium & Medium-High \\
\hline Interest Rate Risk & Medium-High & Medium \\
\hline Abandonment Option & Medium & Medium \\
\hline Liquidity & Medium-High & High \\
\hline
\end{tabular}

Table 1. Categorization of the investments $A$ and $B$.

Based on the fuzzy rules defined by the expert and the linguistic adverbs, the resulting categorization of the investments $A$ and $B$ is the following:

(2) Investment A is Highly Socially-Moderate and Highly Psychologically-Moderate

(3) Investment A is Regular Socially-Moderate and Regular Psychologically-Moderate

The translation of these fuzzy sets and rules to the business portfolio analysis matrix is shown in Figure 13:

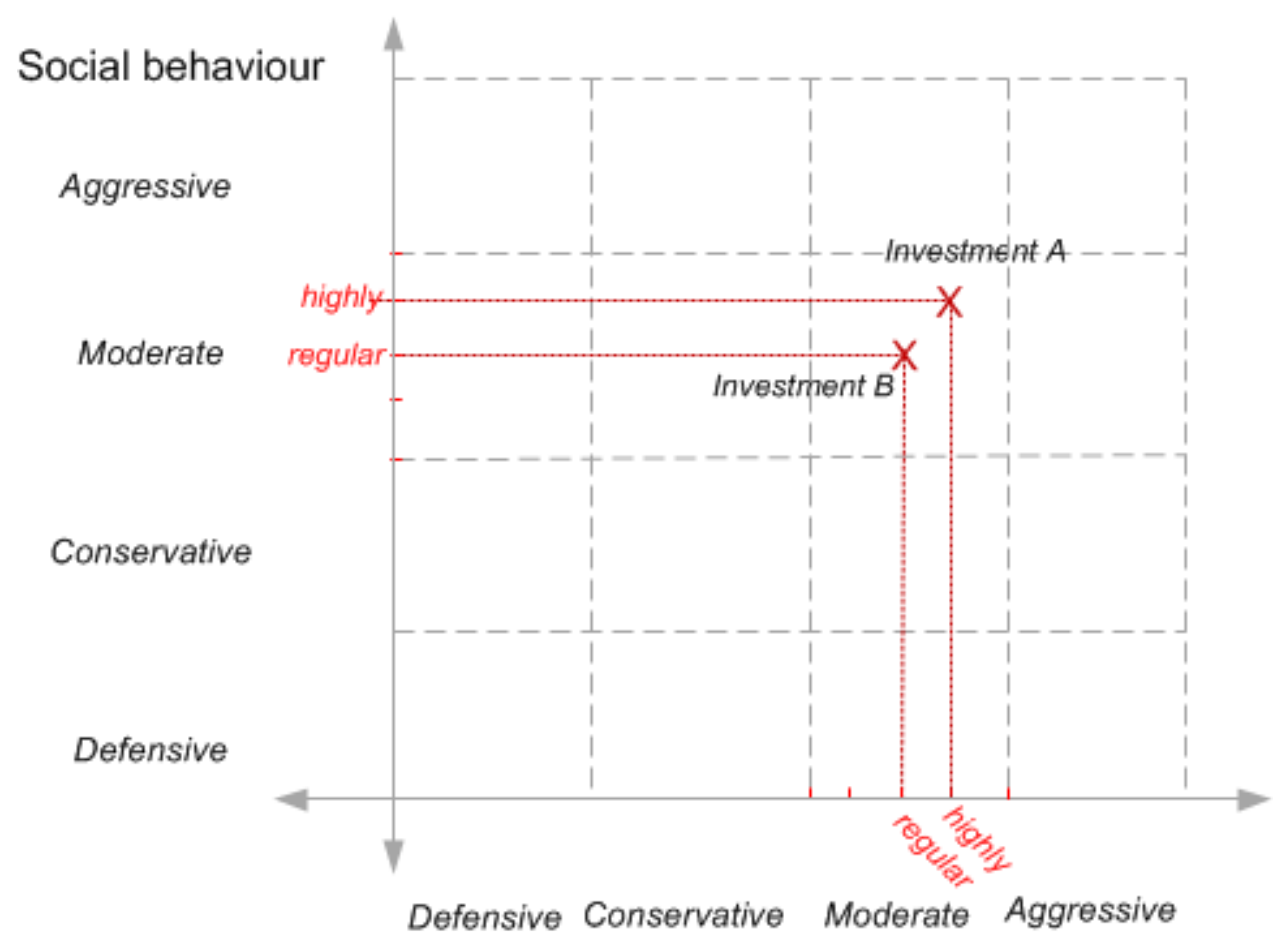

\section{Psychological Behaviour}

Figure 13. Matrix representation of Investments A and B 
Next, the expert has tuned a set of values for the weights used in equation (11), so the following FDs are obtained for the investor and the investments by applying the equations mentioned in the previous section:

- $\mathrm{FD}_{\text {investor }}=(0.26,0.68)$

- $F D_{\text {Investment } \mathrm{A}}=(0.17,0-25)$

- $\mathrm{FD}_{\text {Investment } \mathrm{B}}=(0.12,0-37)$

Finally, to find the recommendation of products portfolio that best fits the characteristics of the investor Peter Smith, three ellipses will be plotted. The first ellipse represents, taking into account the $F D_{\text {investor, }}$, the area of influence of the investor. The other two ellipses represent, based on $\mathrm{FD}_{\text {Investment } A}$ and $F D_{\text {Investment B, }}$, the area of influence of each investment. In Figure 14, these ellipses and the overlapped areas for this example are shown.

\section{Social behaviour}

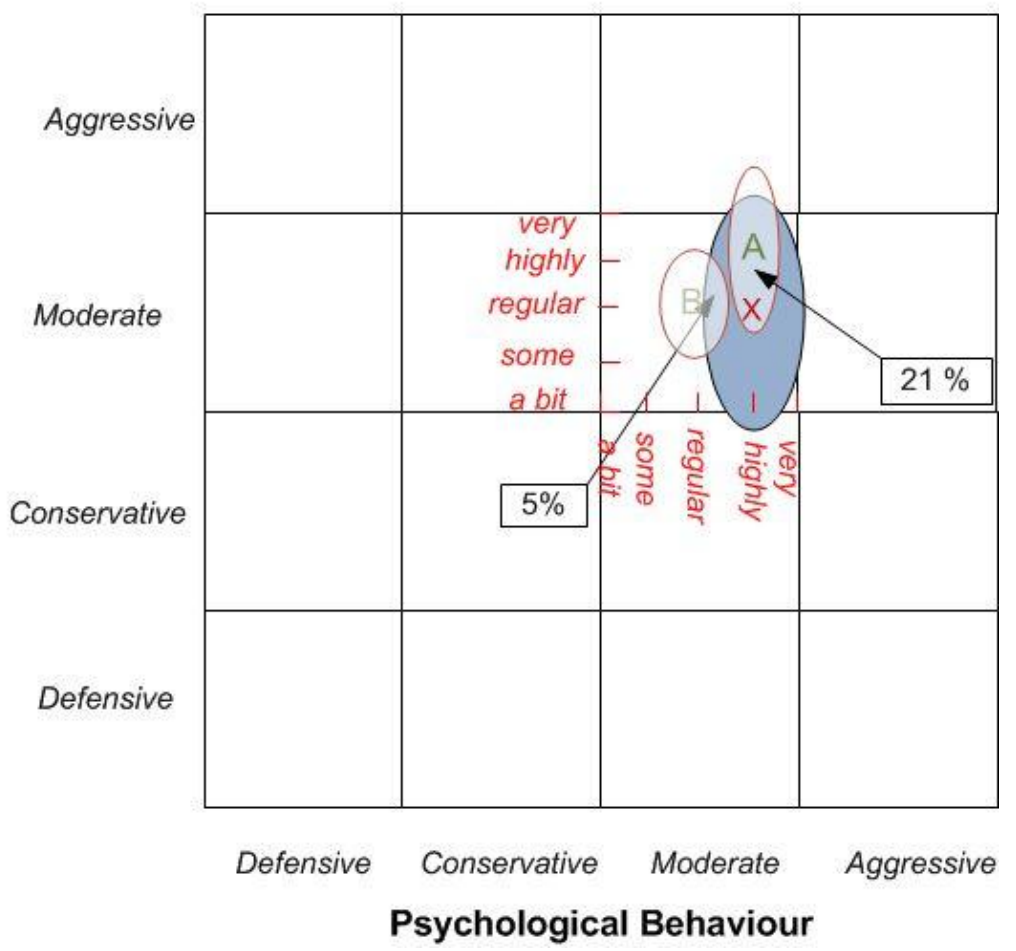

Figure 14. Geometrical recommendation with priority for investments A and B.

In this example the overlapped area of investment A over the area of the investor area is larger than the overlapped area of investment B, so the system will propose the first as the better option for the investor Peter Smith.

\subsection{SINVLIO Architecture}

Figure 13 depicts a three-layer scheme that represents SINVLIO architecture along with its main interactions. 


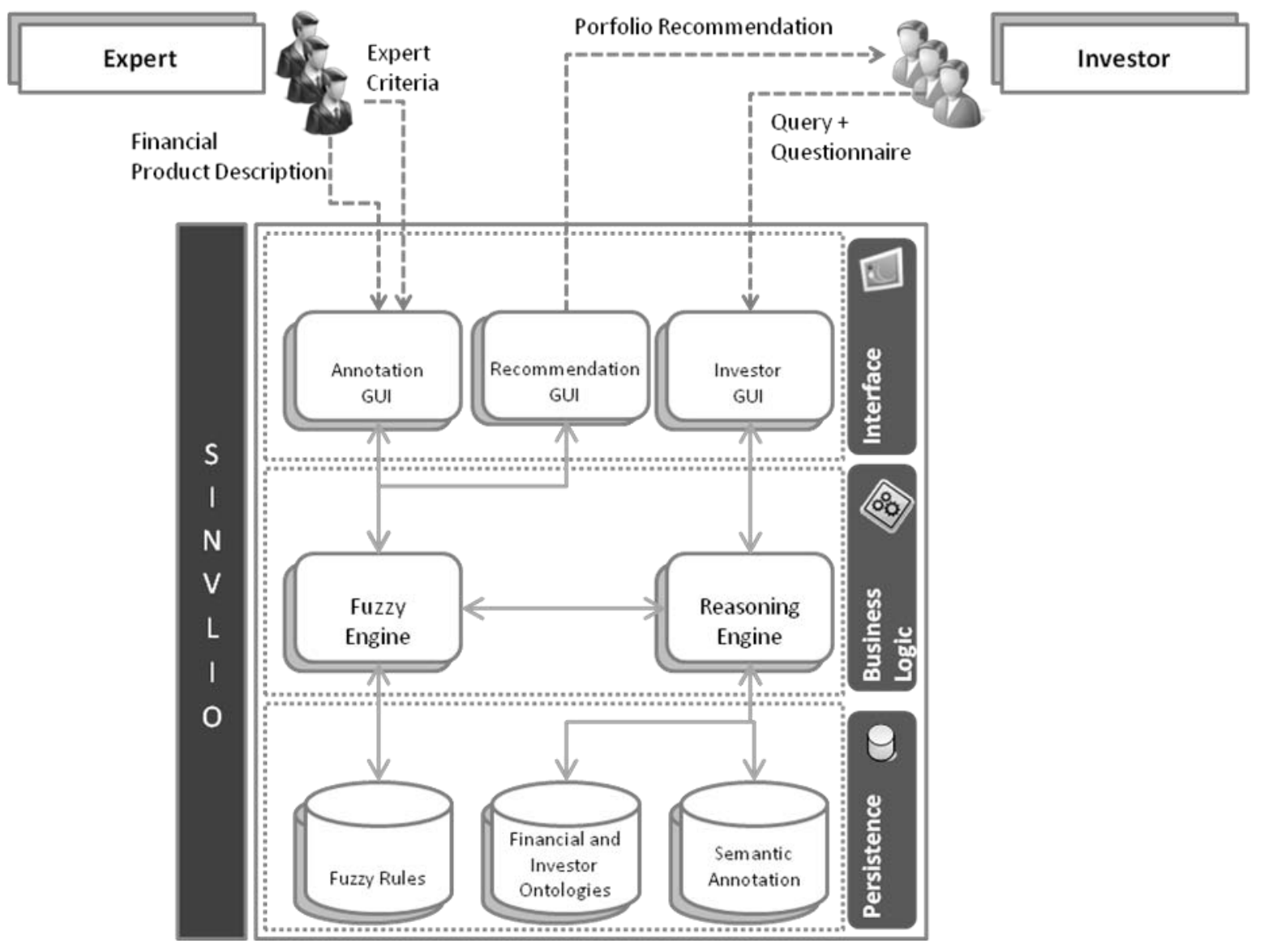

Figure 15. SINVLIO architecture.

The overall functionality of the proposed system is depicted in Figure 15:

1) In the first step, the investor answers a questionnaire about his or her risk preferences and social aspects, as well as particular characteristics of the investment, such as how much money the investor wants to spend.

2) After the questions have been answered, the fuzzy system determines the risk profile of the investor, based on the fuzzy rules previously defined.

3) Once the risk profile of the investor has been established, the fuzzy system determines the characteristics of the financial products in terms of fuzzy expressions.

4) Based on the fuzzy results, the financial products are selected from the product database. These products have been previously described and annotated according to the expert criteria and to the financial ontology.

5) The system generates a set of portfolios based on the selected products and the characteristics of the investment.

6) The portfolios are rated according to their characteristics and they are recommended to the investor.

Before the system can be used, the financial products must be labeled according to the terms of the financial ontology, and the rules for matching the financial products with the investor's risk profile must be defined. 
SINVLIO is structured in three layers (Figure 15):

(I) Interface layer. The user interface consists of three different modules. On the one hand, the annotation GUI allows for the management of financial products, as well as the definition of the fuzzy sets and fuzzy rules that enable the creation of the investment portfolios. This is a web interface which is available in a private area of the developed website. On the other hand, the investor GUI also provides the investor with a questionnaire in order to determine his or her risk profile. Once the questionnaire has been answered, the investor inputs the approximate amount of money $\mathrm{s} / \mathrm{he}$ wants to invest. When all questions have been answered, SINVLIO presents a recommendation regarding the most suitable portfolio in terms of risk tolerance through the Recommendation GUI.

(II) Business logic. The business logic layer consists of a fuzzy engine and a reasoning engine. The fuzzy engine evaluates the defined fuzzy rules in order to determine the investors' risk tolerance. When the investment advisor represents his knowledge by means of the annotation interface, the fuzzy engine validates the fuzzy sets and the fuzzy rules. The semantic information relative to the characteristics of the investors and the investment products is retrieved by the reasoning engine. This reasoning engine has been developed based on the Jena framework. Jena provides Resource Description Framework (RDF) and Ontology Web Language (OWL) support as well as an API for writing and extracting data from OWL descriptions. It is an open source framework that fits with the requirements of the platform proposed in this paper and the research team had experience of this framework.

(III) Persistence layer. This layer stores knowledge of the investor categorization as well as knowledge of the investment products, with the relations between the investment products and the four investment profiles also being taken into account. All the characteristics of the investors and the financial products are defined by means of OWL ontologies. The fuzzy sets and the fuzzy rules are stored into a database. The system's database also stores the relationships between the fuzzy rules and the characteristics represented with the ontologies.

\section{Evaluation}

The subsequent section describes the empirical evaluation of the proposal. The final aim of this study is to determine whether SINVLIO serves as a valid recommendation system in a controlled environment.

\subsection{Evaluation process}

An experimental phase was carried out to check the validity of the proposed recommendation system. This experiment had to take into account expert criteria and the customer profile according to the investor and financial ontologies.

The evaluation was composed of two phases. The aim of phase 1 was to provide a set of test cases in order to execute phase 2. Firstly, a set of investors were asked to fill in the questionnaires defined for the categorization of investors. Secondly, a set of experts would analyze a set of investments offered by Spanish financial entities in order to categorize them 
according to the psychological and social risk profile of their possible investors. Both investor questionnaires and investment categorizations would be processed by SINVLIO in phase 2 . During phase 2, SINVLIO would provide a set of recommendations for the investors identified in phase 1. At the same time, experts also provided a set of investments for the investors in order to compare the results with SINVLIO. The expert recommendations would be carried out by a group of experts using the Delphi method (which allows agreement between the experts' point of view). The selected experts were to fill in a questionnaire in order to categorize the set of sample products. SINVLIO was configured to divide the recommendation in two investment windows in order to diversify the investment risks. The first investment window covered the range of the $0-10,000 €$ of the investment while the second window covered the rest of the amount of the investment.

After obtaining the recommendations of SINVLIO and experts, both windows were compared for further study using standard precision, recall and F1 as well as coverage metrics. Finally a comparison with other related works has been carried out.

\subsection{Sample}

In order to implement the evaluation, there were 25 investors who took part in the experiment (17 men and 8 women with an average age of 32.1 years). These investors were master degree students and teachers at the Universidad Carlos III de Madrid and they had different risk profiles according to their social and psychological characteristics.

For providing recommendations, three kinds of financial products were selected: fixed term deposits, structured deposits and currency deposits. These product families were selected because deposits are an area of high level investment in Spain. A financial expert helped to select 12 products of each kind.

Results were evaluated by four expert investment advisors- with an average of 15 years' experience- from the Spanish private banking sector.

As described in section 4.1, the experiment was carried out in two phases. The next subsection describes the results obtained in each of these phases.

\subsubsection{Phase 1: Categorization of Investors and Investments}

Phase 1 consists of two steps. Firstly, 25 investors were selected in order to participate in this experiment. Each investor filled out the questionnaire designed to determine the psychological and social profiles of risk aversion (as described in section 3 ). These questionnaires were then used in phase 2 to provide investment recommendations according to each investor profile. Table 2 summarizes the investor profiles identified in the study

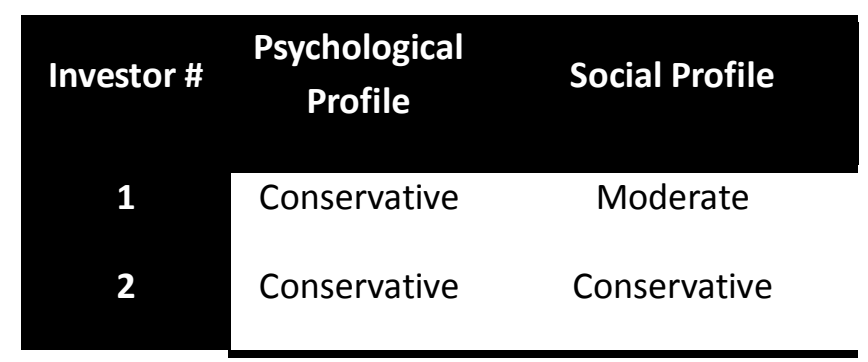




\begin{tabular}{|c|c|c|}
\hline 3 & Conservative & Defensive \\
\hline 4 & Defensive & Conservative \\
\hline 5 & Aggressive & Moderate \\
\hline 6 & Conservative & Moderate \\
\hline 7 & Moderate & Aggressive \\
\hline 8 & Moderate & Moderate \\
\hline 9 & Moderate & Defensive \\
\hline 10 & Defensive & Conservative \\
\hline 11 & Conservative & Conservative \\
\hline 12 & Defensive & Aggressive \\
\hline 13 & Moderate & Aggressive \\
\hline 14 & Conservative & Conservative \\
\hline 15 & Conservative & Moderate \\
\hline 16 & Conservative & Moderate \\
\hline 17 & Defensive & Conservative \\
\hline 18 & Conservative & Conservative \\
\hline 19 & Moderate & Moderate \\
\hline 20 & Aggressive & Moderate \\
\hline 21 & Aggressive & Aggressive \\
\hline 22 & Defensive & Defensive \\
\hline 23 & Conservative & Defensive \\
\hline 24 & Defensive & Aggresive \\
\hline 25 & Defensive & Aggresive \\
\hline
\end{tabular}

Table 2. Investor profiles.

Secondly, 36 products were selected and categorized. As mentioned in the research design section, each product was classified according to the different social and psychological categorization of the potential investor. This classification was carried out by the four experts using the Delphi method. Each expert assigned each product to a square of a grid that represented the most appropriate social and psychological profiles for the product (Figure 16). 


\section{Investment Categorization}

\section{Social behaviour

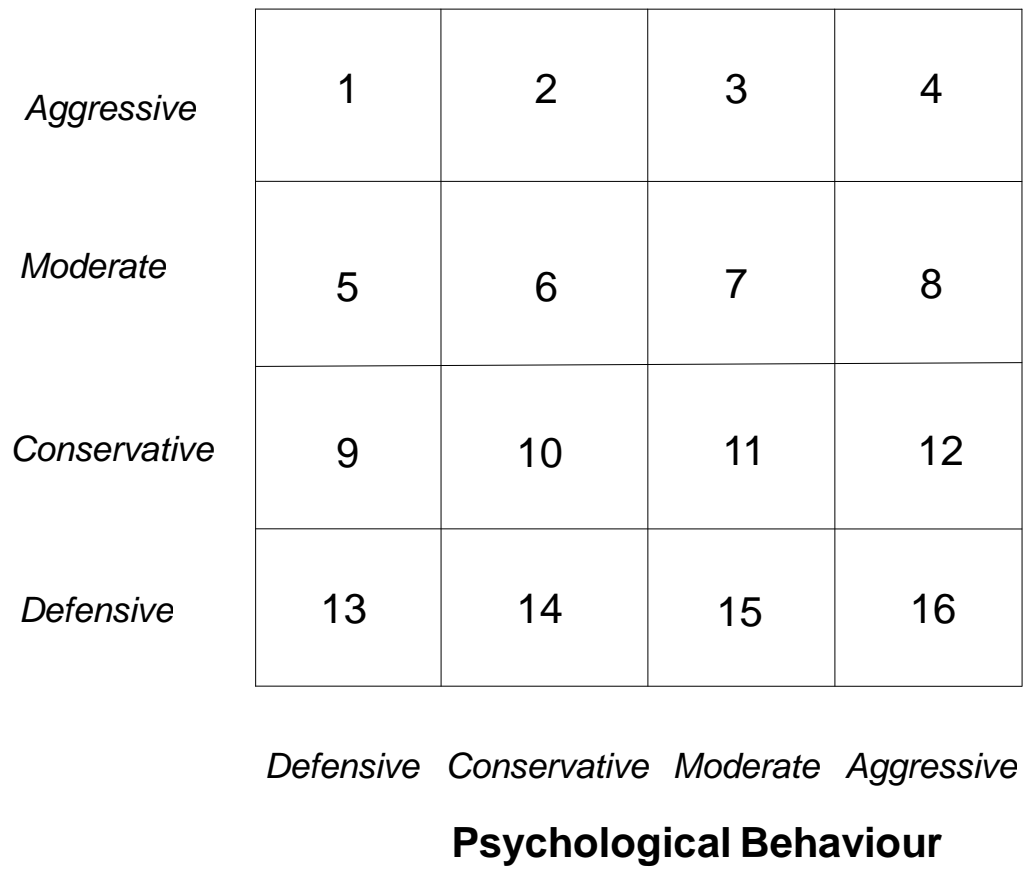

Figure 16. Grid for Delphi categorization of products.

Kendall's coefficient of concordance (W) was computed to measure the level of agreement among the experts. The four experts obtained a coefficient of agreement of $W=0.7938$. Finally, agreement between experts was achieved by applying the Delphi method. The final classification of the products selected for the evaluation is depicted in Figure 17. The classification obtained was used in order to validate the SINVLIO recommendation. The selected products were represented in SINVLIO in order to provide investment recommendations in phase 2 . 


\section{Investment Categorization}

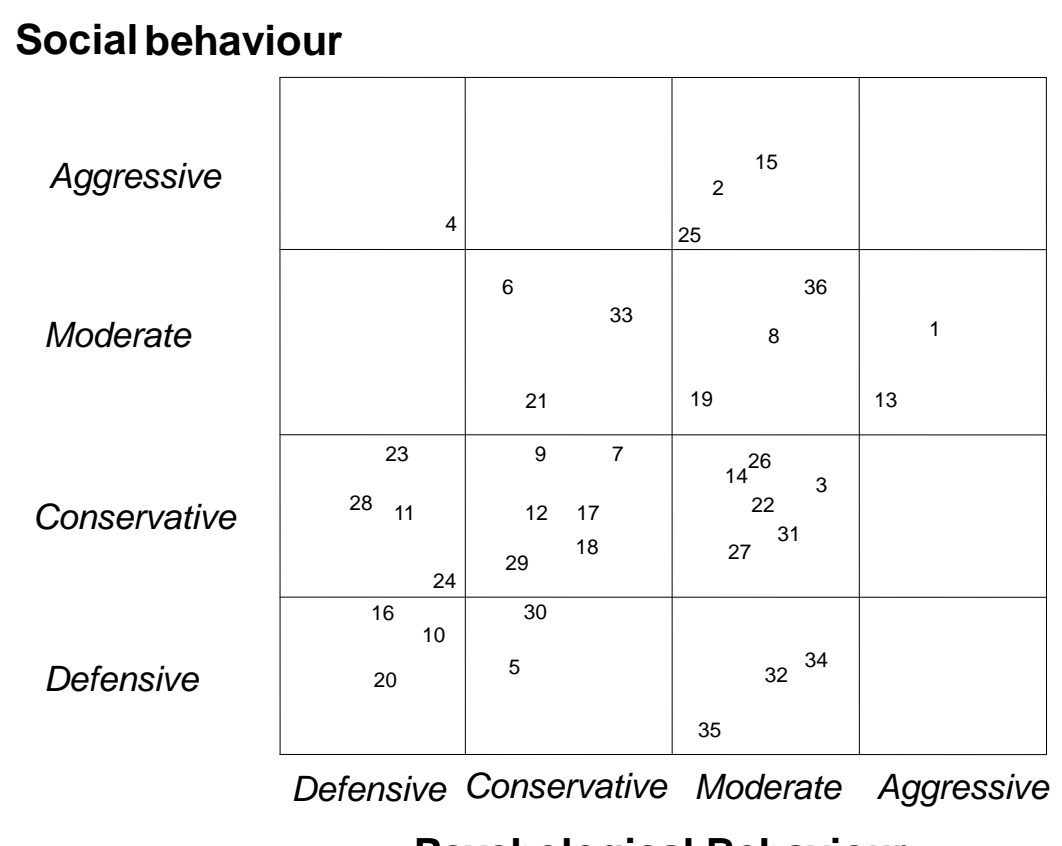

Psychological Behaviour

Figure 17. Product categorization using the Delphi method.

\subsubsection{Phase 2: Recommendations}

Once all of the 36 financial products had been labeled, phase 2 began and a portfolio recommendation was generated based on the risk profile of both investors and financial products. The results of the questionnaires obtained in phase 1 were introduced in SINVLIO and a set of recommendations was obtained for each investor. Simultaneously, based on the same questionnaires and the selected products, the four expert financial advisors made a recommendation to be compared with the results of SINVLIO. Agreement among the experts for the recommendation was achieved using the Delphi method.

As mentioned in the research design, the recommendation was divided into two investment windows in order to diversify the investments. For each investment window SINVLIO recommends two products.

\begin{tabular}{|c|c|c|c|c|c|}
\hline Investor & $\begin{array}{l}\text { Investment } \\
\text { Window }\end{array}$ & $\begin{array}{c}\text { SINVLIO } \\
\text { Recommendation } \\
1\end{array}$ & $\begin{array}{c}\text { SINVLIO } \\
\text { Recommendation } \\
2\end{array}$ & $\begin{array}{c}\text { Delphi } \\
\text { Recommendation } \\
1\end{array}$ & $\begin{array}{c}\text { Delphi } \\
\text { Recommendation } \\
2\end{array}$ \\
\hline 1 & 1 & 9 & 21 & 6 & 21 \\
\hline 1 & 2 & 12 & 17 & 7 & 12 \\
\hline
\end{tabular}




\begin{tabular}{|c|c|c|c|c|c|}
\hline 2 & 1 & 12 & 5 & 17 & 12 \\
\hline 2 & 2 & 5 & 30 & 30 & 5 \\
\hline 3 & 1 & 10 & 5 & 30 & 5 \\
\hline 3 & 2 & 10 & 20 & 16 & 20 \\
\hline 4 & 1 & 20 & 24 & 11 & 24 \\
\hline 4 & 2 & 16 & 20 & 24 & 16 \\
\hline 5 & 1 & 1 & 13 & 1 & 13 \\
\hline 5 & 2 & 3 & 1 & 13 & 3 \\
\hline 6 & 1 & 6 & 21 & 6 & 33 \\
\hline 6 & 2 & 7 & 17 & 21 & 7 \\
\hline 7 & 1 & 15 & 8 & 15 & 25 \\
\hline 7 & 2 & 36 & 19 & 2 & 19 \\
\hline 8 & 1 & 26 & 19 & 8 & 19 \\
\hline 8 & 2 & 14 & 22 & 14 & 27 \\
\hline 9 & 1 & 5 & 32 & 32 & 35 \\
\hline 9 & 2 & 30 & 5 & 30 & 5 \\
\hline 10 & 1 & 16 & 24 & 28 & 24 \\
\hline 10 & 2 & 10 & 20 & 16 & 20 \\
\hline 11 & 1 & 5 & 18 & 17 & 29 \\
\hline 11 & 2 & 5 & 30 & 18 & 5 \\
\hline 12 & 1 & 4 & 23 & 4 & 6 \\
\hline 12 & 2 & 23 & 28 & 6 & 21 \\
\hline 13 & 1 & 2 & 36 & 2 & 25 \\
\hline 13 & 2 & 36 & 8 & 36 & 19 \\
\hline 14 & 1 & 30 & 12 & 17 & 29 \\
\hline 14 & 2 & 5 & 30 & 29 & 5 \\
\hline
\end{tabular}




\begin{tabular}{|c|c|c|c|c|c|}
\hline 15 & 1 & 7 & 21 & 33 & 21 \\
\hline 15 & 2 & 29 & 17 & 12 & 29 \\
\hline 16 & 1 & 6 & 33 & 6 & 33 \\
\hline 16 & 2 & 9 & 12 & 21 & 9 \\
\hline 17 & 1 & 11 & 24 & 28 & 24 \\
\hline 17 & 2 & 28 & 16 & 11 & 24 \\
\hline 18 & 1 & 12 & 30 & 17 & 12 \\
\hline 18 & 2 & 5 & 30 & 30 & 5 \\
\hline 19 & 1 & 14 & 19 & 8 & 19 \\
\hline 19 & 2 & 14 & 31 & 14 & 27 \\
\hline 20 & 1 & 1 & 13 & 1 & 13 \\
\hline 20 & 2 & 3 & 1 & 13 & 3 \\
\hline 21 & 1 & 25 & 36 & 36 & 15 \\
\hline 21 & 2 & 8 & 19 & 36 & 8 \\
\hline 22 & 1 & 16 & 20 & 16 & 10 \\
\hline 22 & 2 & 10 & 20 & 10 & 20 \\
\hline 23 & 1 & 16 & 10 & 29 & 10 \\
\hline 23 & 2 & 10 & 20 & 5 & 10 \\
\hline 24 & 1 & 4 & 16 & 4 & 6 \\
\hline 24 & 2 & 16 & 10 & 6 & 33 \\
\hline 25 & 1 & 4 & 23 & 4 & 6 \\
\hline 25 & 2 & 23 & 28 & 6 & 21 \\
\hline
\end{tabular}

Table 3. SINVLIO Recommendations vs. Expert Recommendations

Table 3 shows the recommendations provided by SINVLIO for each investor and window, and the agreed expert recommendation using Delphi method. In order to determine the accuracy of the SINVLIO results, Precision and Recall and F1 measures were used to measure the degree of relevance of the recommendations provided by SINVLIO with respect to the experts' recommendation. The following describes several perspectives regarding the implementation of this analysis. 
Firstly, the products recommended by SINVLIO were compared with the products recommended by the experts. In this first analysis, the products recommended by the experts were considered correct recommendations. If the product recommended by SINVLIO coincides with that recommended by the experts (the first product of the first investment window in both cases) then it was considered a correct investment found. Precision value determines the number of correct products offered by SINVLIO with respect to the overall products offered by SINVLIO. Recall value represents the relation between the number of correct products offered by SINVLIO with respect to the overall correct products (in this first analysis the products selected by the expert). The formulae applied for the calculations are:

$$
\begin{gathered}
\text { precision }=\frac{\text { CorrectInvestmentsFound }}{\text { TotalInvestmentFound }} \\
\text { recall }=\frac{\text { CorrectInvestmentsFound }}{\text { TotalCorrectInvestments }} \\
F_{1}=\frac{2 * \text { precision } * \text { recall }}{\text { precision }+ \text { recall }}
\end{gathered}
$$

\begin{tabular}{|cccc|}
\hline $\begin{array}{c}\text { Overall SINVLIO Recommendations vs. Overall } \\
\text { expert Recommendations }\end{array}$ & 0.32 & 0.32 & Precision \\
\hline $\begin{array}{c}\text { First SINVLIO Recommendation for each window } \\
\text { vs. First expert recommendation for each window }\end{array}$ & 0.3 & 0.3 & 0.3 \\
\hline $\begin{array}{c}\text { Second SINVLIO Recommendation for each } \\
\text { window vs. Second expert Recommendation for } \\
\text { each Window }\end{array}$ & 0.34 & 0.34 & 0.34 \\
\hline
\end{tabular}

Table 4. Precision and Recall analysis for product recommendations.

Table 4 shows the values obtained in the first precision and recall study. Precision and recall values are the same because the number of correct recommendations is the same as the number of recommendations offered by SINVLIO.

At this moment, the recommendations are considered correct when SINVLIO and the experts provided the same order for the same window. Table 5 shows the precision and recall results for each SINVLIO recommendation with respect to the two products offered by the expert for the same investment window. In this particular case, it's important to note that precision and recall scores are different, since correct investments are not equal to suggested investments. In this new scenario, F1 values of the whole recommendation and the first recommendation are higher than those shown in Table 4.

\begin{tabular}{|cccc|}
\hline & Precision & Recall & F1 \\
\hline $\begin{array}{c}\text { SINVLIO Recommendation vs. Expert } \\
\text { Recommendations for each window }\end{array}$ & 0.51 & 0.255 & 0.34 \\
\hline $\begin{array}{c}\text { First SINVLIO Recommendation for each window vs. } \\
\text { Experts recommendations for each window }\end{array}$ & 0.6 & 0.3 & 0.4 \\
\hline $\begin{array}{c}\text { Second SINVLIO Recommendation for each window } \\
\text { vs. Experts Recommendations for each Window }\end{array}$ & 0.42 & 0.21 & 0.28 \\
\hline
\end{tabular}


Table 5. Precision and Recall values for each window

After analyzing the agreement between the products offered by SINVLIO and the products selected by the experts, a second study based on precision and recall was performed. This second study consisted of comparing the product categorization selected by SINVLIO with that selected by the experts. For example, if the first product recommended by SINVLIO was categorized by the experts as socially moderate and psychologically moderate (cell 7 in Figure 16) and the product offered by the expert was also classified in the same way, then this was considered a correct result.

\begin{tabular}{|cccc|}
\hline Overall SINVLIO Categorization vs. Overall expert & Precision & Recall & F1 \\
\hline $\begin{array}{c}\text { Categorization } \\
\text { First SINVLIO Categorization for each window vs. First } \\
\text { expert Categorization for each window }\end{array}$ & 0.6 & 0.6 & 0.48 \\
\hline $\begin{array}{c}\text { Second SINVLIO Categorization for each window vs. } \\
\text { Second expert Categorization for each Window }\end{array}$ & 0.72 & 0.72 & 0.48 \\
\hline
\end{tabular}

Table 6. Precision and Recall results for product categorization

Table 6 shows the values of precision and recall related to the product categorization offered by SINVLIO and compares them to the same categorization offered by the experts. The expert categorization is considered as the correct categorization. Thus, if SINVLIO and the experts provide a result from the same category, we consider it to be a correct value.

The above-mentioned results show the values of precision and recall with respect to the expert recommendation. However the precision of SINVLIO can be measured with respect to the products of the categories recommended by the expert. For example if the expert proposes investments from the category Moderate-Conservative, the precision and recall is calculated considering all the products categorized as Moderate-Conservative to be correct investments. In this case, the difference with the results shown in Table 6 is the number of correct values in the number of categories proposed by the experts, while in table 7 , the number of correct values is the number of investments of the experts' categorization. Table 7 shows the results obtained for the described precision and recall measures considering the results for each window investment.

\begin{tabular}{|cccc|}
\hline SINVLIO Recommendation vs. Investments in the & Precision & Recall & F1 \\
\hline $\begin{array}{c}\text { correct categorization } \\
\text { First SINVLIO Recommendation for each window vs. } \\
\text { Investments in the categorization of first the expert } \\
\text { recommendation for each window }\end{array}$ & 0.6 & 0.195 & 0.294 \\
\hline $\begin{array}{c}\text { Second SINVLIO Recommendation for each window vs. } \\
\text { Investments in the categorization of first the expert } \\
\text { recommendation for each window }\end{array}$ & 0.72 & 0.193 & 0.276 \\
\hline
\end{tabular}

Table 7. Precision and Recall results for investments with correct categorization

The values of Precision and Recall are the mean of the precision and recall values for each recommendation of SINVLIO. Table 8 presents the results obtained if the set of correct 
products is extended to all products categorized in both categories determined by the expert for each investment window.

\begin{tabular}{|lccc|}
\hline & Precision & Recall & F1 \\
\hline $\begin{array}{c}\text { SINVLIO Recommendation vs. Investments in the } \\
\text { categorizations of the expert recommendations for the } \\
\text { window }\end{array}$ & 0.76 & 0.241 & 0.366 \\
\hline $\begin{array}{c}\text { First SINVLIO Recommendation vs Investments in the } \\
\text { categorizations of the expert recommendations for the } \\
\text { window }\end{array}$ & 0.74 & 0.278 & 0.405 \\
\hline $\begin{array}{c}\text { Second SINVLIO Recommendation vs. Investments in the } \\
\text { categorizations of the expert recommendations for the } \\
\text { window }\end{array}$ & 0.78 & 0.25 & 0.379 \\
\hline
\end{tabular}

Table 8. Precision and recall results for recommendations in categories proposed by the experts

The second dimension in testing is Coverage. The coverage of a system is a measure of the domain of items in the system over which the system can form predictions or make recommendations. This measure can be calculated as the percentage of items for which a recommendation system can provide recommendations. The easiest way to measure coverage in our case is to select a random sample of user/product pairs, ask for a prediction for each pair, and measure the percentage for which a prediction was provided [37]. A high coverage value indicates that the recommendation system provides assistance in selecting among most of the items while a low coverage means that the system may be less valuable to users, since they will be limited in decision making capacity [60]. In this case, taking into account that SINVLIO provides two recommendations, Table 9 includes Coverage results of SINVLIO comparing the first of the options only and both options:

\begin{tabular}{|l|r|r|}
\cline { 2 - 3 } \multicolumn{1}{c|}{} & SINVLIO First Result & SINVLIO Couple of Results \\
\hline Coverage & 0.417 & 0.778 \\
\hline
\end{tabular}

Table 9. SINVLIO Coverage results

\subsection{Discussion}

On analyzing the results presented in section 4.2 , it can be seen that $32 \%$ of products offered by SINVLIO are the same as those recommended by the experts. This is logical, taking into account the number of products, the distance between the products in the representation and the subjectivity of the estimators. When not considering the order of the recommendation, the precision value increased up to $51 \%$. These results may be regarded as satisfactory.

However, these good marks could be very restrictive because they consider that the only valid recommendation is that proposed by the experts. As shown in section 4.2., we have analyzed the results which consider valid values those products of the categories recommended by the expert (i.e. if the expert recommends an investment categorized as Conservative-Moderate, then all possible investments classified as Conservative-Moderate form the set of valid investments). In this case (see Table 7), the precision value increases up to $60 \%$, although $\mathrm{F} 1$ is nearly 0.3 in all cases. This indicates that the system is able to approximate the product category in order to offer a suitable result. On analyzing the results of the system compared with the customer profile, it can be observed that the system tends to be more conservative in 
classifying the social profile than experts. Furthermore, a study of the results shows that the second option provided by the experts is, in general, more conservative. It explains the fact that the second product offered by the system fits better than the first product recommended by experts ( $48 \%$ vs. $72 \%$ ). If in this case, the order of the recommendation is not considered, the precision value increases to $76 \%$. As mentioned before, taking into account the subjectivity of the recommendation process and the characteristics of the investments, the results were considered as accurate by the experts. Thus, a high degree of similarity was obtained between SINVLIO and the experts' recommendations.

The comparison of SINVLIO with previous efforts in the literature throws good results for this tool. Focusing on efforts tested using Precision, Recall and F1 metrics, the effectiveness of such metrics in recommender systems assessment has been proven by several authors in different domains [23]. Thus, although there are not efforts devoted to portfolio recommendation tested using these metrics, there are a panoply of works to provide a remarkable testing set.

Table 10 shows the comparison of SINVLIO results with a set of recent and relevant initiatives related to recommendation:

\begin{tabular}{|clrrr|}
\hline System & Domain & Precision & Recall & F1 \\
\hline SINVLIO & Portfolio & 0.32 & 0.32 & 0.32 \\
\hline$[\mathbf{8 4}]$ & Conversion Rates & 0.097 & 0.291 & 0.139 \\
\hline$[\mathbf{8 5}]$ & eCommerce & 0.238 & 0.091 & 0.131 \\
\hline$[\mathbf{2 4}]$ & Tourism & 0.48 & 0.48 & 0.48 \\
\hline$[47]$ & Music & 0.241 & 0.439 & 0.311 \\
\hline$[62]$ & Digital Libraries & 0.675 & 0.613 & 0.635 \\
\hline$[61]$ & Digital Libraries & 0.511 & 0.677 & 0.566 \\
\hline$[48]$ & Knowledge Management Systems & 0.728 & 0.663 & 0.688 \\
\hline
\end{tabular}

Table 10. Comparison of SINVLIO metrics with relevant literature.

Results show that SINVLIO recommendation accuracy is remarkable. Focusing on F1 metric, there are works reaching more than 0.6 points, which is an outstanding mark. However, it's important to underline that the complexity of the domain makes SINVLIO notable. Although digital libraries and knowledge management systems are complex scenarios, the truth is that expert investment recommendations are not easy to achieve using soft computing means. On the one hand, the volatility of the expert judges and facts and on the other hand the level of complex operations needed to classify both investors and investments are factors that affect the recommendation. Then, the comparison of SINVLIO with similar environments (e.g. [84]) places the tool presented in this paper in a good position. Not in vain, F1 metric in SINVLIO reaches 0.32 while [84] reaches 0.139 .

It is also important to underline that SINVLIO uses a set of information that roots on the psychological and social characteristics of the investor. Thus, preferences are based on beliefs and other characteristics of the investor. In [47], authors deal with similar characteristics. It this case, the compassion of SINVLIO and [47] produce comparable results (0.32 vs. 0.311 ). 
With respect to coverage metrics, one more time there are recent and relevant initiatives that test recommender systems using this mean (e.g. [60], [27], [67]). As stated before, there are not works appliying this metric in the field of application of SINVLIO. However we can see that SINVLIO results is in the line of [67], where results peek 0.65, provides better results than [27], [37], where coverage are around 0.38 and are less than [59]. In this latter case, where coverage slightly less than perfect for typical level of sparsity (Coverage: 99\%), the dataset is more convenient to produce good results.

In conclusion, results reveal a good performance of the proposed system and encourage authors to improve SINVLIO and to apply the knowledge acquired in its development in new environments and tools.

\section{Conclusions and Future Work}

This paper presents SINVLIO, a tool that extends the concept of portfolio selection enounced by Markowitz [54] by including a combination of two different points of view: the psychological and the social characteristics of the investors. The main goal of this research is to provide a behavioral orientation to portfolio recommendations by means of fuzzy logic and semantic technologies. Taking into account the findings of many previous works, this paper has proposed a method in which both investors and investments are defined and classified by ontology and are then matched by applying fuzzy technologies.

In order to provide recommendations, three kinds of financial products were selected: fixed term deposits, structured deposits and currency deposits. These product families have been selected because deposits are an area of high level investment in Spain. The evaluation was conducted with the help of a financial expert who assisted in selecting 12 examples of each type of product. The results obtained were analyzed with the collaboration of four expert investment advisors- with an average of 15 years' experience- from the Spanish private banking sector. The results have been defined as very accurate, with a high degree of similarity between SINVLIO and the expert recommendations. Different possibilities have been analyzed, such as whether or not to take into account the order of the recommendation, and the results which consider valid values those products of the categories recommended by the expert have been studied. For all these reasons, the results have been considered reliable, considering the subjective aspects of the decision-making of the experts and the degree of similarity between products.

The current paper proposes two types of initiatives which should be explored in future research. Firstly, our future work plans to study the distances between the products recommended by SINVLIO and the products recommended by experts. Next, the authors will expand the features of SINVLIO in order to provide a ranking of recommended investments instead of a pair of recommendations, and test this new feature using metrics such as Mean average precision and Mean reciprocal rank.

\section{Acknowledgements}

This work is supported by the Spanish Ministry of Industry, Tourism, and Commerce under the projects SONAR2 (TSI-020100-2008-665) and GO2 (TSI-020400-2009-127). 


\section{References}

[1] Abiyev, R. H. and Menekay, M. Fuzzy Genetic System for Modelling Investment Portfolio. In PRICAI 2006: Trends in Artificial Intelligence. Lecture Notes in Computer Science, vol. 4099/2006, 2006, pp. 701-710.

[2] Adomavicius, G., and Tuzhilin, A. Toward the next generation of recommender systems: A survey of the state-of-the-art and possible extensions. IEEE Transactions on Knowledge and Data Engineering, 17(6) (2005), pp. 734-749.

[3] Baker, H. K. and Nofsinger, J. R. Psychological Biases of Investors. Financial Services Review, 11 (2002), pp. 97-116.

[4] Beach, S.L. and Rose, C.C. Does Portfolio Rebalancing Help Investors Avoid Common Mistakes? Journal of Financial Planning, 18 (5) (2005), pp. 56-61.

[5] Cabrerizo, F.J., Pérez, I.J. and Herrera-Viedma, E. Managing the consensus in group decision making in an unbalanced fuzzy linguistic context with incomplete information. Knowledge-Based Systems, 23 (2) (2010), pp. 169-181.

[6] Chang Chien, Y.W., Chen, Y. L. Mining associative classification rules with stock trading data - A GA-based method, Knowledge-Based Systems, 23 (6) (2010), pp. 605-614.

[7] Chen, C. T. and Hung, W. Z. A New Decision-Making Method for Stock Portfolio Selection Based on Computing with Linguistic Assessment. Journal of Applied Mathematics and Decision Sciences, (2009), Article id: 897024. doi:10.1155/2009/897024.

[8] Chen, J. S., Hou, J. L., Wua, S. M. and Chang-Chien, Y. W. Constructing investment strategy portfolios by combination genetic algorithms. Expert Systems with Applications, 36 (2) (2009), pp. 3824-2828.

[9] Chen, M.C., Lin, C.L. and Chen, A.P. Application of XCSR Model for Dynamic Portfolio Selection. Contemporary Management Research, 5 (1) (2009), pp. 67-76.

[10]Cheng, H., Lu, Y. and Sheu C. An ontology-based business intelligence application in a financial knowledge management system. Expert Systems with Applications, 36 (2, Part 2) (2009), pp. 3614-3622.

[11]Cho, V. MISMIS - A comprehensive decision support system for stock market investment, Knowledge-Based Systems, 23 (6) (2010), pp. 626-633.

[12]Cohn, R. A., Lewellen, W. G., Lease, R. C. and Schlarbaum, G. C. Individual Investor Risk Aversion and Investment Portfolio Composition. The Journal of Finance, 30 (2) (1975), pp. 605-620.

[13]Corter, J.E. and Chen, Y.J. Do investment risk tolerance attitudes predict portfolio risk? Journal of Business and Psychology, 20 (3) (2006), pp. 369-381. 
[14] Cox, E., Fuzzy logic and the measures of certainty in eCommerce expert systems. PC Al, 15 (3) (2001), pp. 16-22.

[15] Da Silva Borges, P., A model of strategy games based on the paradigm of the Iterated Prisoner's Dilemma employing Fuzzy Sets. PhD Thesis. Universidade Federal de Santa Catarina, Production and Systems Engineering, 1996.

[16]Daniel, K., Hirshleifer, D. and Teoh, S.H. Investor psychology in capital markets: evidence and policy implications. Journal of Monetary Economics, 49 (1) (2002), pp. 139-209.

[17] David, F.R., Strategic Management, Prentice-Hall, Upper Saddle River, NJ, 2001.

[18]De Bondt, W., Muradoglu, G., Shefrin, H. and Staikouras, S. K. Behavioral Finance: Quo Vadis?. Journal of Applied Finance, 18 (2) (2008), pp. 1-21.

[19]Dichev, I.D. and Janes, T.D. Lunar cycle effects in stock returns. Journal of Private Equity, 6 (4) (2003), pp. 8-29.

[20]Dimova, L., Sevastianov, P. and Sevastiano, D., MCDM in a fuzzy setting: investment projects assessment application, International Journal of Production Economics, 100 (1) (2006), pp. 10-29.

[21]Fellner, G. and Maciejovsky, B. Risk attitude and market behavior: Evidence from experimental asset markets. Journal of Economic Psychology, 28 (3) (2007), pp. 338350.

[22]García-Crespo, A., Colomo-Palacios, R., Gómez-Berbís, J.M. and García-Sánchez, F. SOLAR: Social Link Advanced Recommendation System. Future Generation Computer Systems, 26 (3) (2009), pp. 374-380.

[23] García-Crespo, A., Colomo-Palacios, R., Gómez-Berbís, J.M. and Ruiz-Mezcua, B. SEMO: a framework for customer social networks analysis based on semantics. Journal of Information Technology, 25 (2) (2010), pp. 178-188.

[24] García-Crespo, Á., López-Cuadrado, J.L., Colomo-Palacios, R., González-Carrasco, I. \& Ruiz-Mezcua, B. Sem-Fit: A semantic based expert system to provide recommendations in the tourism domain. Expert Systems with Applications, 38(10) (2011), 13310-13319.

[25]García-Manotas, I., Lupiani, G., García-Sánchez, F. and Valencia-García, R. Populating Knowledge Based Decision Support Systems. International Journal of Decision Support System Technology, 2 (1) (2010), pp. 1-20.

[26] Ghazinoorya, S., Zadehb, A. E. and Kheirkhahb, A. S. Application of fuzzy calculations for improving portfolio matrices. Information Sciences, 180 (9) (2010), pp. 1582-1590.

[27]Göksedef, M., Gündüz-Öğüdücü, Ş. Combination of Web page recommender systems. Expert Systems with Applications, 37(4) (2010), 2911-2922

[28] Grable, J. E. Risk Tolerance. In: Xiao, J. J. (ed) Handbook of Consumer Finance Research, Springer, New York, 2008, pp. 3-19. 
[29] Grable, J. E., Financial risk tolerance and additional factors that affect risk taking in everyday money matters, Journal of Business and Psychology, 14 (4) (2000), pp. 625630.

[30]Grable, J.E. and Lytton, R. H., Financial risk tolerance revisited: the development of a risk assessment instrument, Financial Services Review, 8 (3) (1999), pp. 163-181.

[31]Grable, J.E. and Lytton, R. H., The development of a Risk Assessment Instrument: A Follow-Up Study, Financial Services Review, 12 (3) (2003), pp. 257-274.

[32] Gupta P., Mehlawat M. K. and Saxena A. A hybrid approach to asset allocation with simultaneous consideration of suitability and optimality. Information Sciences, 180 (11) (2010), pp. 2264-2285.

[33]Hadavandi, E., Shavandi, H. and Ghanbari, A. Integration of genetic fuzzy systems and artificial neural networks for stock price forecasting, Knowledge-Based Systems, 23 (8) (2010), pp. 800-808.

[34] Hambrick, D., I. MacMillan, and D. Day. Strategic attributes and performance in the BCG matrix: A PIMS-based analysis of industrial-product businesses. Academy of Management Journal, 25 (3) (1982), pp. 510-531.

[35] Harlow W. V. and Brown K. C. Understanding and Assessing Financial Risk Tolerance: A Biological Perspective. Financial Analysts Journal, 46 (6) (1990), pp. 50-62.

[36] Hawley, D. D., Johnson, J. D. and Raina, D. Artificial Neural Systems: A New Tool for Financial Decision-Making. Financial Analysts Journal, 46 (6) (1990), pp. 63-72.

[37]Herlocker, J.L., Konstan, J.A., Terveen, L.G., \& Riedl, J.T. Evaluating Collaborative Filtering Recommender Systems. ACM Transactions on Information Systems, 22(1) (2004), 5-53.

[38]Hilton, D.J. The Psychology of Financial Decision-Making: Applications to Trading, Dealing, and Investment Analysis. Journal of Behavioral Finance, 2 (1) (2001), pp. 3753.

[39]Hirshleifer, D. Investor psychology and asset pricing. Journal of Finance, 56 (4) (2001), pp. 1533-1597.

[40]Huang, X. Portfolio selection with a new definition of risk. European Journal of Operational Research, 186 (1) (2008), pp. 351-357.

[41]Hui, E.C.M., Lau, O.M.F. and Lo, K.K. A fuzzy decision-making approach for portfolio management with direct real estate investment. International Journal of Strategic Property Management, 13 (2) (2009), pp. 191-204.

[42]IFIP. The Financial Exchange Framework Ontology. International Financial Information Publishing Ltd (IFIP) (2003). http://www.financial-format.com/fef.htm. Last access: March 11, 2010 
[43]Kahneman, D. and Riepe, M. W. Aspects of Investor Psychology . Beliefs, preferences and biases investment advisors should know about. Journal of Portfolio Management, 24 (4) (1998), pp. 1-22.

[44]Keller, C. and Siegrist, M. Money Attitude Typology and Stock Investment. Journal of Behavioral Finance, 7 (2) (2006), pp. 88-96.

[45]Konana, P. and Balasubramanian, S. The Social-Economic-Psychological model of technology adoption and usage: an application to online investing, Decision Support Systems, 39 (3) (2005), pp. 505-524.

[46]Kuo, M.H., Chen, L.C. and Liang, C.W. Building and evaluating a location-based service recommendation system with a preference adjustment mechanism. Expert Systems with Applications, 36 (2, Part 2) (2009), pp. 3543-3554.

[47]Lee, S.K., Cho, Y.H., \& Kim, S.H. Collaborative filtering with ordinal scale-based implicit ratings for mobile music recommendations. Information Sciences, 180(1) (2010), 21422155.

[48]Li, M., Liu, L., \& Li, C.B. An approach to expert recommendation based on fuzzy linguistic method and fuzzy text classification in knowledge management systems. Expert Systems with Applications, 38(7) (2011), 8586-8596.

[49]Liang, T.P., Lai, H.J., and Ku, Y.C. Personalized content recommendation and user satisfaction: theoretical synthesis and empirical findings. Journal of Management Information Systems, 23(3) (2006), pp. 45-70.

[50]Lighthall, N.R., Mather, M. and Gorlick, M.A. Acute Stress Increases Sex Differences in Risk Seeking in the Balloon Analogue Risk Task. PLoS ONE, 4 (7) (2009). doi:10.1371/journal.pone.0006002.

[51]Liu, J., Wang, W., Golnaraghi, F. and Kubica, E. A neural fuzzy framework for system mapping applications. Knowledge-Based Systems, 23 (6) (2010), pp. 572- 579.

[52]Mamdani, E. H. and Assilian, S. An experiment in linguistic synthesis with a fuzzy logic controller. International Journal of Human-Computer Studies, 51 (2) (1999), pp. 135147.

[53]Mamdani, E. H. Fuzzy sets and applications: selected papers by L. A. Zadeh: R R Yager, S Ovchinikov, R M Tong, H T Nguyen (eds). Knowledge-Based Systems, 1 (2) (1988), pp. 121-121.

[54]Markowitz, H. Portfolio selection. Journal of Finance, 7 (1) (1952), pp. 77-91.

[55]Muhammad, N. M. N. Behavioral Finance Vs Traditional Finance. Advances in Management, 2 (6) (2009), pp. 1-10.

[56]Nanda, S.R., Mahanty, B., and Tiwari, M.K. Clustering Indian stock market data for portfolio management. Expert Systems with Applications, 37 (12) (2010), pp. 87938798. 
[57]Negnevitsky, M. Artificial Intelligence: A Guide to Intelligent Systems, Addison-Wesley Longman Publishing Co., Inc., Boston, MA, 2005.

[58]Niininen, O., Buhalis, D. and March, R. Customer empowerment in tourism through consumer centric marketing (CCM). Qualitative Market Research: An International Journal, 10(3) (2007), pp. 265-281.

[59]Papagelis, M., \& Plexousakis, D. Qualitative analysis of user-based and item-based prediction algorithms for recommendation agents. Engineering Applications of Artificial Intelligence, 18(7) (2005), 781-789.

[60]Park, Y.J., \& Chang, K.N. Individual and group behavior-based customer profile model for personalized product recommendation. Expert Systems with Applications, 38(2, Part 1) (2009), 1932-1939.

[61]Porcel, C., López-Herrera, A.G., \& Herrera-Viedma, E. A recommender system for research resources based on fuzzy linguistic modeling. Expert Systems with Applications, 36(1, Part 1) (2009), 5173-5183.

[62]Porcel, C., and Herrera-Viedma, E. Dealing with incomplete information in a fuzzy linguistic recommender system to disseminate information in university digital libraries. Knowledge-Based Systems, 23 (1) (2010), pp. 32-39.

[63]Qin, Z., Li, X. and Ji, X. Portfolio selection based on fuzzy cross-entropy. Journal of Computational and Applied Mathematics, 228 (1) (2009), pp. 139-149.

[64]Quek, C., Yow, K.C., Cheng, P.Y.K. and Tan, C.C. Investment portfolio balancing: application of a generic self-organizing fuzzy neural network (GenSoFNN). Intelligent Systems in Accounting Finance and Management, 16 (1) (2009), pp. 147-164.

[65]Ricci, F. and Werthner, H. Recommender systems. International Journal of Electronic Commerce, 11(2) (2006), pp. 5-9.

[66]Roscoe, P. and Howorth C. Identification through technical analysis: A study of charting and UK non-professional investors. Accounting, Organizations and Society, 34 (2) (2009), pp. 206-221.

[67]Samizadeh, R., \& Ghelichkhani, B. Use of semantic similarity and web usage mining to alleviate the drawbacks of user-based collaborative filtering recommender systems use. International Journal of Industrial Engineering and Production Research, 21(3) (2010), 137-146.

[68]Shefrin, H. and Statman, M. Behavioral Portfolio Theory. Journal of Financial and Quantitative Analysis, 35 (2) (2000), pp. 127-151.

[69]Shipley, M. F. Portfolio Management: A Fuzzy Set Based Approach to Monitoring Size to Maximize Return and Minimize Risk. World Academy of Science, Engineering and Technology, 52 (2009), pp. 506-513. 
[70]Shyng, J.Y., Shieh, H.M., Tzeng, G.H. and Tzeng, S.H. Using FSBT technique with Rough Set Theory for personal investment portfolio analysis. European Journal of Operational Research, 201 (2) (2010), pp. 601-607.

[71]Shyng, J.Y., Shieh, H.M., Tzeng, G.H. and Tzeng, S.H., An integration method combining Rough Set Theory with formal concept analysis for personal investment portfolios, Knowledge-Based Systems, 23 (6) (2010), pp. 586-597.

[72]Skinner, B.F. About behaviorism. Alfred A. Knopf, New York, 1974.

[73]Statman, M. Normal Investors, Then and Now. Financial Analyst Journal, 61 (2) (2005), pp. 31-36.

[74]Studer, R., Benjamins, V.R. and Fensel, D. Knowledge engineering: Principles and methods. Data and Knowledge Engineering, 25 (1/2) (1998), pp. 161-197.

[75]Sudebar, Z., McCrae, M. and Gerace, D. The Validity of Financial Advisor's Heuristic Risk Tolerance Categorisation: Evidence From a Risk Tolerance Assessment Tool. In: European Financial Management Association 2007 Annual Meetings, 2007.

[76] Thompson, A.A.; Strickland, A.J., Strategic Management Concepts and Cases, IRWIN Homewood, Boston, 1992.

[77]Valentine, J. L. Applying Expert Systems To Investment. Financial Analysts Journal, 44 (6) (1988), pp. 48-53.

[78]Watson, J. B. Psychology as the behaviorist views it. Psychological Review, 20 (1913), pp. 158-177.

[79]Wong, F. S., Wang, P. Z., Goh, T. H. and Quek, B. K. Fuzzy Neural Systems for Stock Selection. Financial Analysts Journal, 48 (1) (1992), pp. 47- 53.

[80] Xie G, Xiong R, Church I. Comparison of kinetics, neural network and fuzzy logic in modelling texture changes of dry peas in long time cooking. Lebensmittel-Wissenschaft und-Technologie, no. 31 (7-8) (1998), pp. 639-647.

[81]Yan, L. Risk Curve and Bifuzzy Portfolio Selection. Journal of Mathematics Research, 1 (2) (2009), pp. 193-198.

[82]Yang, B., Li, L.X., Ji, H., Xu, J. An early warning system for loan risk assessment using artificial neural networks, Knowledge-Based Systems, 14 (5-6) (2001), pp. 303-306

[83]Zadeh, L. Fuzzy sets. Information and Control, 8 (3) (1965), pp. 338-353.

[84]Zanker, M. \& Jessenitschnig, M. Case-studies on exploiting explicit customer requirements in recommender systems, User Modeling and User-Adapted Interaction, 19 (2009) 133-166.

[85]Zanker, M., Jessenitschnig, M. \& Schmid, W. Preference reasoning with soft constraints in constraint-based recommender systems, Constraints, 15 (2010) 574-595. 